\title{
MIMO Fading Emulator Development with FPGA and Its Application to Performance Evaluation of Mobile Radio Systems
}

\author{
Yoshio Karasawa, Katsuhiro Nakada, Guijiang Sun, and Rikako Kotani \\ Advanced Wireless and Communication Research Center (AWCC), The University of Electro-Communications (UEC), \\ 1-5-1 Chofugaoka, Chofu, Tokyo 182-8585, Japan \\ Correspondence should be addressed to Yoshio Karasawa; karasawa@ee.uec.ac.jp
}

Received 18 January 2017; Revised 23 May 2017; Accepted 6 June 2017; Published 13 July 2017

Academic Editor: Miguel Ferrando Bataller

Copyright (C) 2017 Yoshio Karasawa et al. This is an open access article distributed under the Creative Commons Attribution License, which permits unrestricted use, distribution, and reproduction in any medium, provided the original work is properly cited.

\begin{abstract}
We present four new developments for a multiple-input multiple-output (MIMO) over-the-air measurement system based on our previous studies. The first two developments relate to the channel model for multipath environment generation. One is a further simplification of the circuit configuration without performance degradation by reducing the number of delay generation units, which dominate the performance limit when implementing the circuit on a field-programmable gate array (FPGA). The other is to realize spatial correlation characteristics among the input ports on the transmission side, whereas the previously proposed channel model did not consider this correlation. The third development involves the details of implementing the MIMO fading emulator on an FPGA as a two-stage scheme. The fourth is the demonstration of application examples of the developed system.
\end{abstract}

\section{Introduction}

In recent years, as the speed and capacity of information communication technology have grown, multiple-input multipleoutput (MIMO) transmission schemes have become a key technology for mobile radio systems. Effective evaluation of MIMO communication terminal performance in actual radio wave environments has been obtained in over-the-air (OTA) measurements, in which the device under test (DUT) is placed in a specifically constructed fading environment [1]. Various means of constructing MIMO-OTA measurement systems have been proposed and discussed in the 3rd Generation Partnership Project (3GPP) and other forums for the standardization of mobile communications [2].

Of these MIMO-OTA measurement systems, one type is a field emulation system, which is used to produce a multipath fading environment around the DUT by surrounding the terminal with multiple probe antennas [3-9]. Conventional OTA measurement systems, as typified in [5], have the benefit of generating an environment based on mature technologies used in commercially available fading simulators. However, the systems proposed so far result in large and complicated configurations for MIMO-OTA applications.
In this context, we have directed our research toward simplification of the circuitry to emulate the fading environment $[10,11]$. In $[10]$, we proposed a new circuit configuration named "antenna-branch-controlled type." In [11], we used computer simulation of this proposed system to clarify the MIMO channel characteristics as a function of the number of probe antennas. In $[12,13]$, we reported an implementation of the signal-processing unit on a field-programmable gate array (FPGA).

When measuring DUT performance using a MIMOOTA system, it is necessary to construct a complicated experimental setup in a large radio anechoic chamber with a number of probe antennas. To simplify these measurements, a two-stage method has been proposed [14]. In the two-stage method, the DUT's antenna radiation pattern is measured in the first stage, and the actual performance evaluation is carried out by incorporating the measured antenna pattern data into the second-stage emulation. We used computer simulation to verify the functionality of this two-stage method in a configuration incorporating our proposed antenna-branchcontrolled scheme [15].

In this paper, we present four new developments for a MIMO-OTA measurement system based on our previous 


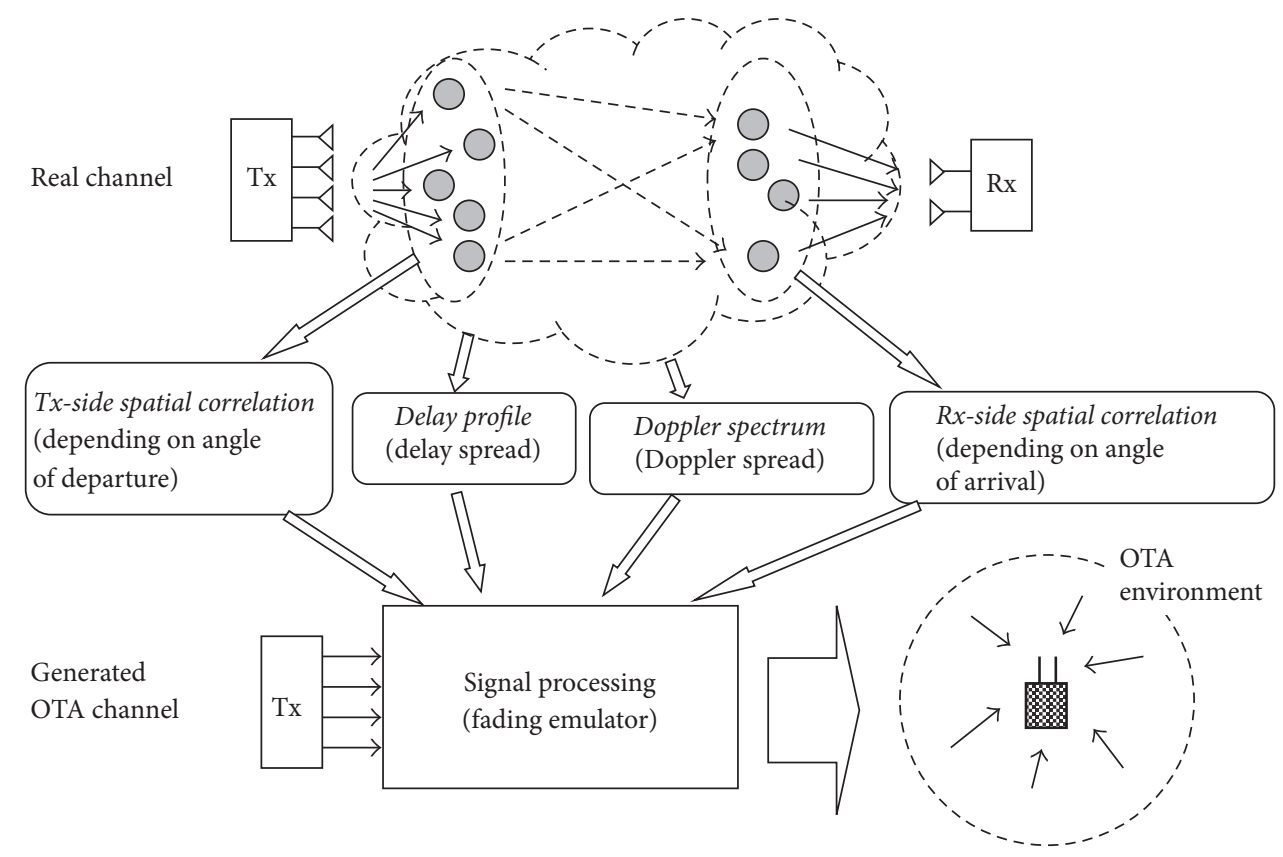

FIGURE 1: Triply selective multipath environment and its emulation.

studies $[10-13,15]$. The first two relate to the channel model for multipath environment generation. One development is a further simplification of the circuit configuration without performance degradation by reducing the number of delay generation units, which dominate the performance limit when implementing the circuit on an FPGA. The other is to realize spatial correlation characteristics among input ports at the transmission side, whereas our previous channel model [11] did not consider this correlation. The third development is implementation of the proposed system on an FPGA incorporating the two-stage scheme, and the fourth is to show application examples of the developed system.

\section{Brief Summary of MIMO-OTA Testing Systems}

2.1. Basic Functions for OTA Measurements. In OTA testing, a realistic propagation environment is generated around the receiving terminals for measurement of transmission performance characteristics. For OTA system development, the multipath propagation channel models proposed thus far by various researchers [16-20] require the following functions: (i) transmission (TX) side spatial correlation, (ii) Doppler spread, (iii) delay spread, and (iv) reception (RX) side spatial correlation. The angular spread (i and iv), Doppler spread (ii), and delay spread (iii) cause space-, time-, and frequencyselective fading, respectively, forming a so-called "triply selective" multipath environment. Figure 1 shows how these selectivities fit into the overall MIMO-OTA measurement system. Further details on MIMO-OTA systems and their standardization works can be found in [2].

In OTA test methods, the environment for MIMO terminal evaluation is generated by either a reverberation chamber $(\mathrm{RC})$ or a fading emulator $(\mathrm{FE})[2,21]$. In the $\mathrm{RC}$ system, a chamber with metallic walls that effectively reflect radio waves is used to generate a rich multipath propagation environment [22-24]. In FE systems, a number of virtual scattering probe antennas encircle DUTs to generate a fading environment [3-9, 25-29]. FE systems can provide flexible control of propagation parameters and various propagation environments, but system construction is complex and costly. RC systems lack flexibility in propagation parameter control but can readily provide a multipath-rich environment over a wide range of frequencies. To incorporate the benefits of both FE and RC, a hybrid OTA testing scheme has been proposed [30]. Although both methods have advantages and limitations, this paper focuses on FE OTA systems.

2.2. FE OTA Systems. Figure 2 shows a basic configuration of an FE MIMO-OTA measurement system. The overall system comprises three parts: the transmitting antenna ports $(M$ ports), the probe antennas ( $L$ antennas), and the receiving antenna ports ( $N$ ports) of the terminal (DUT), together with the multipath channel generation part that links the input ports and the probe antennas.

When designing an OTA system, the number of delayed paths $K$ gives the performance limit because they consume circuit resources significantly. Figure 3 shows two typical configurations in the delay generation part. Figure 3(a) shows the conventional configuration, the "path-controlled type," which uses $M L K$ delay units. Almost all existing systems are of this type. Figure 3(b) shows our previously proposed "antenna-branch-controlled type" [11], which uses $L K$ units. Although this configuration is extremely simple, the performance for creating a triply selective multipath environment is basically the same as for the path-controlled configuration. In the latter section in this paper, we propose a further simplified configuration, named "advanced antenna-branch-controlled 


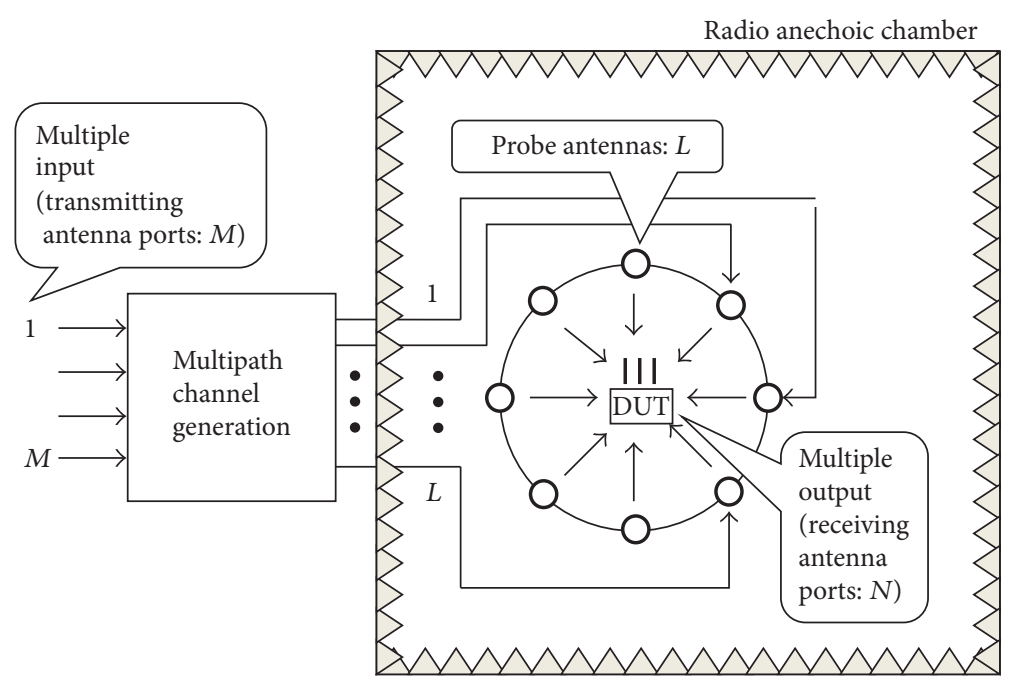

FIgUre 2: General configuration of an FE MIMO-OTA system.

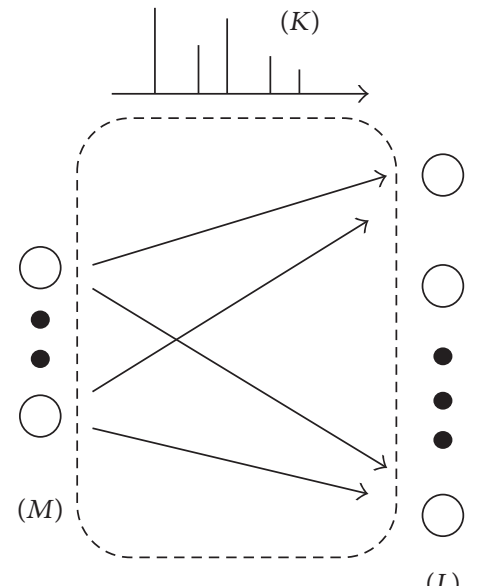

[Delay units: $M L K]$

(a) Path-controlled type

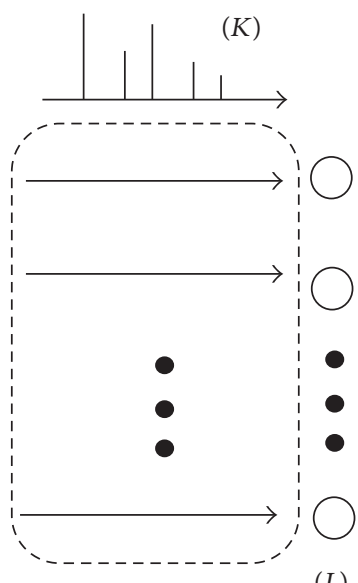

[Delay units: $L K]$

(b) Antenna-branch-controlled type

FIgURE 3: Configurations for delay generation.

type," where the requirement for $L K$ delay units is reduced to $M K$.

2.3. Two-Stage Method. Construction of the measurement system shown in Figure 2 in a radio anechoic chamber is generally tedious work. Moreover, if the DUT is too large, for example, antennas installed in a vehicle [29], the area required to arrange the probe antenna array around the DUT is particularly large and may exceed the size of a conventional radio anechoic chamber. Even in these cases, it is possible to measure antenna array radiation patterns if the distance required for measurements is available in one direction by emitting radio waves from that direction only while rotating the DUT. These needs have recently led to the proposal of a two-stage method, with measurement of the antenna characteristics in the first stage followed by their input into an FE for performance evaluation in the second stage [14, 30, 31].

Figure 4 shows the two-stage measurement scheme. The basic configuration is shown in Figure 4(a), while an image of the environment generated in the second stage is shown in Figure 4(b). Since the two-stage scheme does not radiate any radio waves during fading emulation, it would be better to call this the "MIMO fading emulator (MIMO-FE)," rather than an OTA measurement scheme.

As stated above, a large benefit of the two-stage scheme is the simplicity of the system configuration and DUT performance evaluation. The largest difficulty is a requirement for connecting test cables directly to the antenna ports for the antenna pattern measurement during the first stage. Cabled connection to a very small terminal, such as a 


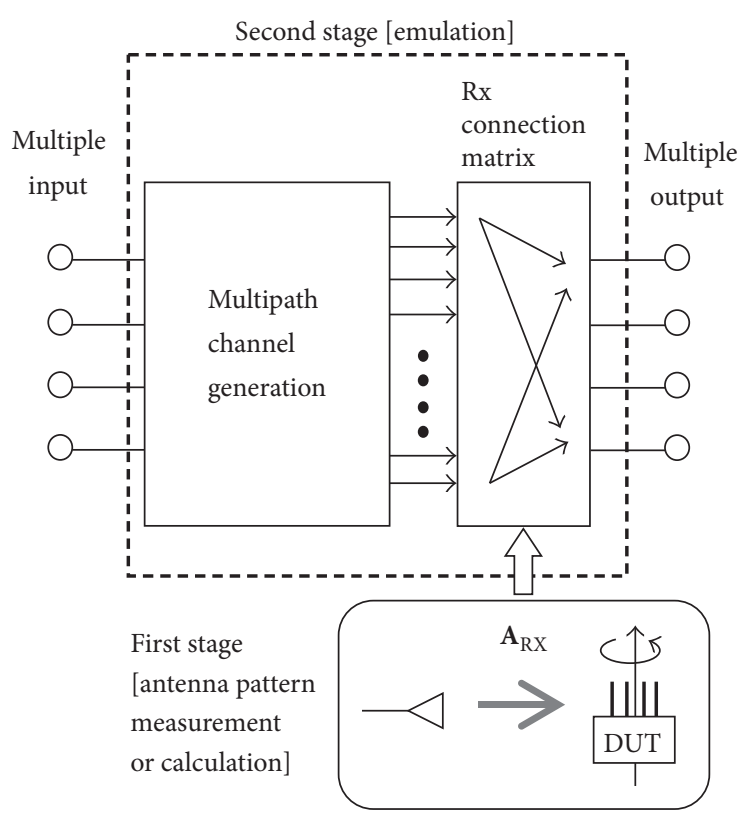

(a) Configuration

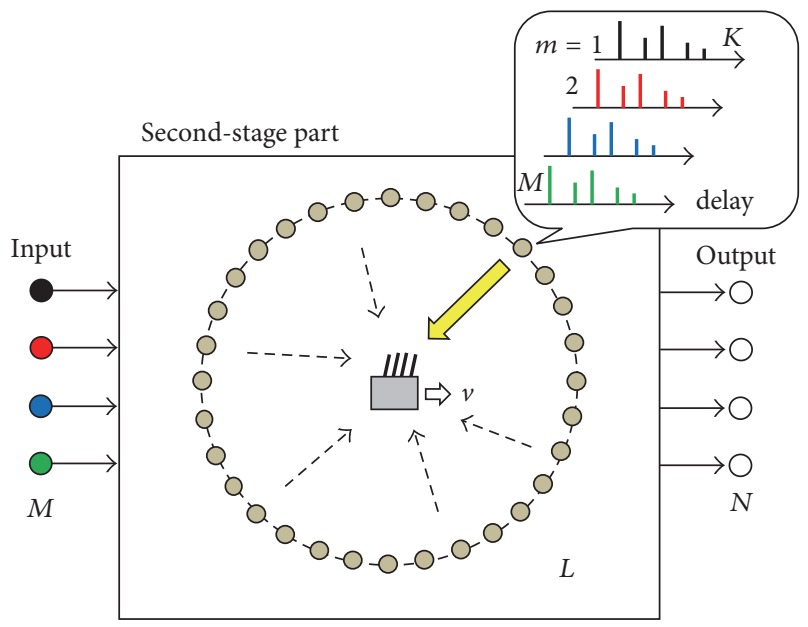

(b) Environment generated in the second stage

FIGURE 4: Two-stage fading emulation scheme.

smartphone, is not appropriate, since the existence of the cable will interfere with the device antenna pattern. For this purpose, a special data collection scheme without disturbing the true antenna patterns has to be incorporated into the DUT. Although there are some countermeasures for this problem [30, 31], it remains generally difficult. Nevertheless, if numerically calculated antenna patterns are available, for example, the performance evaluation can be done very easily by incorporating them into the second-stage emulation.

\subsection{FPGA-Based MIMO-FE. When developing OTA fading} emulators, it has been common to adopt well-developed fading simulator techniques [5], but this has led to large-scale, high-cost systems. A system configuration using an FPGA is promising for making the fading generation part small and light-weight. Table III of [32] summarizes the performance comparisons of various FPGA-based prototypes. As of 2010, the system proposed in [32] realized $N M K<160$ and reached the best performance. The FPGA this system used was an Altera Stratix III, EP3SL150F.

In recent years, a MIMO configuration using generalpurpose software-defined radio transceivers made up of FPGAs has been developed $[33,34]$. These systems provide a $2 \times 2$ configuration, and the delay remains within $1 \mu$ s. On the other hand, we had implemented a MIMO-OTA system with $M=4, L=8, K=8$, and a maximum delay of $200 \mu \mathrm{s}$, based on the antenna-branch-controlled method in Figure 3(b) on an FPGA board [13]. The FPGA used was a Xilinx Virtex-6, LX 240T, which is the same FPGA used in Section 4.

When implementing more complicated circuits on an FPGA, devising the circuit configuration is the key for enhancing function and performance against the limited gate array resources.
2.5. Background on the Novelty of the Developed System. The early stage of FE-type MIMO-OTA developments was mainly focused on control of the direction of arrival (DOA) of radio waves around the terminal by using many probe antennas with 7 to 31 elements $[3,4,6]$. This makes it possible to create Rayleigh fading environments with Doppler frequency variations and RX-side spatial correlation characteristics. However, at this stage, incorporation of delayed wave generation units capable of controlling the delay profile exceeding $1 \mu \mathrm{s}$ was not achieved. On the other hand, in the fading simulator for evaluating the transmission performance of single-input single-output (SISO) systems, a multipath fading link having necessary functions, such as Rayleigh and/or Nakagami-Rice amplitude fluctuations with variable Doppler power spectrum and delay profile, has been available at that time. By applying the technology cultivated in this fading simulator development, an extremely sophisticated FE-type MIMO-OTA measurement system was proposed in [5] based on the path-controlled configuration shown in Figure 3(a) composed of $M L$ links. The developed system based on this scheme has played an important role in standardization of DUT performance measurement schemes [2]. However, since the fading generation function must be created using $M L$ links individually, the system configuration results in large scale and costly. For this reason, more simplification of the system configuration by enhancing the circuit efficiency of multipath generation part in $L \times M$ matrix is required from a practical viewpoint.

In addition, the transmission side spatial correlation characteristics, namely, the distribution of the path directions in the base station side as shown in Figure 1, have not been incorporated explicitly in the $L \times M$ matrix of developed systems available so far. 
Our group, on the other hand, has proposed an antennabranch-controlled method as shown in Figure 3(b) that can realize a MIMO-OTA measurement system with a simple configuration retaining the necessary functions [10, 11]. Particularly in [11], we provide detailed configuration and operation principle of the antenna-branch-controlled scheme and evaluate the accuracy of generated propagation environments by computer simulation. The paper [13] deals with a hardware implementation of the method of [11] using an FPGA and realizing the probe antennas of $L=8$. On the other hand, [14] proposes a configuration of the antenna-branch-control method with the two-stage method and confirms the validity through computer simulation.

The novelty of this paper based on the above-mentioned previous works is summarized as follows:

(i) Further simplification of the configuration of the signal-processing unit, that is, the "advanced antennabranch-controlled method": this scheme can drastically reduce the number of delay units in the $L \times M$ connection matrix, which achieves $L=32$ probe antennas with 8 delay paths varying up to $200 \mu \mathrm{s}$

(ii) Incorporation of the TX-side spatial correlation property to realize the propagation characteristics around the base station site

(iii) Implementation of two-stage MIMO-FE on an FPGA incorporating the functions of (i) and (ii) above

(iv) Evaluation and demonstration of effectiveness in a wireless local area network (WLAN) and terrestrial digital broadcasting reception.

\section{Advanced Channel Model for MIMO Fading Emulator}

3.1. Basic Configuration. We pursued a multipurpose propagation channel model with a triply selective multipath environment where parameters such as direction of arrival, delay profile, and maximum Doppler frequency can be controlled flexibly. In that way, existing MIMO propagation channel models, such as 3GPP SCM, COST 259/273, and Winner [35], can be generated by setting the parameter values as requested.

In the simplified antenna-branch-controlled system [11], the multiple required functions of the multipath channel generator shown in Figure 3(b) are apportioned and connected in a cascade configuration. The spatially uncorrelated feature of the transmission signals is produced using Walsh-Hadamard code sets; then multipath delayed waves are produced for each signal, and finally a different Doppler shift is added for each probe antenna. Rayleigh fading is thus obtained by spatial synthesis of the signals emitted from the probe antennas in a configuration for amplitude and delay control for each path in the connection matrix that is far simpler than conventional path-control methods.

$N \times M$ MIMO transmission and reception signals of $M$ transmitter antennas and $N$ receiver antennas can be expressed as follows:

$$
\mathbf{r}(t)=\mathbf{H}_{\mathrm{FE}}(t, \tau) * \mathbf{s}(t)+\mathbf{n}(t),
$$

where $\mathbf{s}$ is the transmission signal vector ( $M$ dimensions) given as a function of time $t, \mathbf{n}$ is the noise vector $(N$ dimensions), $\mathbf{r}$ is the reception signal vector ( $N$ dimensions), and $\mathbf{H}_{\mathrm{FE}}$ represents the impulse response matrix $(N \times M$ dimensions) of channels described as a function of time $t$ and delay time $\tau$. In the equation, the symbol $*$ represents a convolutional integral. The antenna-branch-control method [11] provides a simpler configuration for channel generation by conducting signal processing in branch units of $L$ probe antennas that create a multipath environment. $\mathbf{H}_{\mathrm{FE}}$ is given as follows:

$$
\mathbf{H}_{\mathrm{FE}}(t, \tau)=\mathbf{A}_{\mathrm{RX}} \mathbf{A}_{\text {Doppler }}(t) \mathbf{H}_{\text {delay }}(\tau) \mathbf{W}_{\mathrm{TX}},
$$

where $\mathbf{W}_{\mathrm{TX}}$ is a TX-side connection matrix with $L \times M$ dimensions consisting of a set of Walsh-Hadamard code vectors, with the matrix producing independent Rayleigh fluctuations against $M$ input signals, and $\mathbf{A}_{\mathrm{RX}}$ is the $N \times$ $L$ channel matrix connecting the probe antenna array with the reception antenna array. For conventional OTA testing systems, $\mathbf{A}_{\mathrm{RX}}$ is automatically determined by the arrangement of both probe antennas and the DUT receiver array without taking receiver array patterns into account. In the case of two-stage systems, all necessary data for $\mathbf{A}_{\mathrm{RX}}$ are obtained during the first-stage measurement of the antenna patterns in a radio anechoic chamber. In (2), $\mathbf{A}_{\text {Doppler }}$ represents an $L \times L$ diagonal matrix with diagonal elements of $\exp \left(j 2 \pi f_{D l} t\right)$ $(l=1,2, \ldots, L)$ that provide the Doppler shifts corresponding to the angular positions $\theta_{l}$ of $L$ probe antennas. Each Doppler shift $f_{D l}$ is given by

$$
f_{D l}=\frac{v}{\lambda} \cos \left(\theta_{l}+\Delta \theta_{l}\right)
$$

where $v$ is the vehicular velocity and $\lambda$ is the wavelength of the radio wave. The small angular offset $\Delta \theta_{l}$ gives spectral variety [11].

3.2. Advanced Antenna-Branch-Controlled Scheme. When implementing the circuit on an FPGA, increasing the number of delay generation units mainly decreases performance because they consume gate resources significantly. As stated in the previous section, the antenna-branchcontrolled scheme in Figure 3(b) realizes fairly large circuitscale reduction compared with the path-controlled scheme in Figure 3(a). Now, we propose the "advanced antenna-branchcontrolled" type shown in Figure 5, which uses only $M K$ delay units. Considering values of $M=4$ and $L=32$, the reduction ratio for the number of necessary units for the new configuration is $1 / 32$ for the scheme in Figure 3(a) and 1/8 for the scheme in Figure 3(b). Here we present the channel model for the proposed configuration.

3.2.1. TX Connection Matrix. The TX connection matrix $\mathbf{W}_{\mathrm{TX}}$ with $M \times M$ dimensions provides specific spatial correlation characteristics for the signals from the $M$ input ports. Since the determination of this matrix is one of the key developments reported in this paper, a detailed description is provided in Section 3.3. The input signal vector $\mathbf{s}(t)$ and the associated output signal vector $\mathbf{s}^{\prime}(t)$ are linked by

$$
\mathbf{s}^{\prime}(t)=\mathbf{W}_{\mathrm{TX}} \mathbf{s}(t) \text {. }
$$




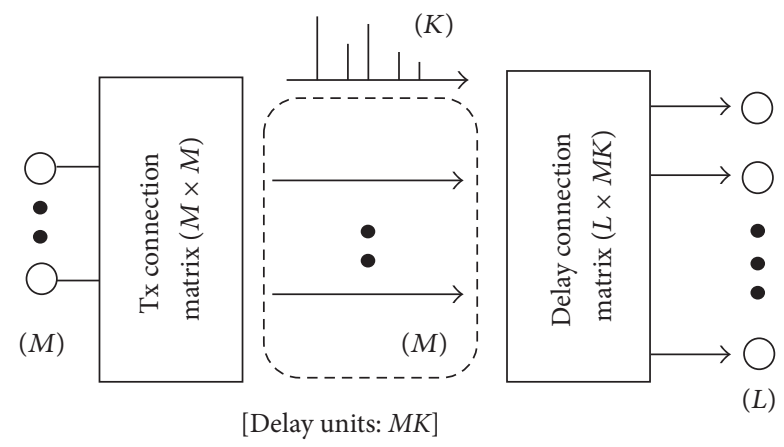

Figure 5: Advanced antenna-branch-controlled scheme.

3.2.2. Delayed Wave Generation. To generate delayed signals for each of the $K$ delayed waves from the above outputs, we construct an $M \times K$ delayed wave matrix:

$$
\begin{aligned}
\mathbf{U}(t) & =\left(\begin{array}{llll}
\mathbf{u}_{1} & \mathbf{u}_{2} & \cdots & \mathbf{u}_{K}
\end{array}\right)=\mathbf{s}^{\prime}(t) * \boldsymbol{\delta}(t) \\
& =\left(\begin{array}{ccc}
s_{1}^{\prime}\left(t-\tau_{1}\right) & \cdots & s_{1}^{\prime}\left(t-\tau_{K}\right) \\
\vdots & \ddots & \vdots \\
s_{M}^{\prime}\left(t-\tau_{1}\right) & \cdots & s_{M}^{\prime}\left(t-\tau_{K}\right)
\end{array}\right)
\end{aligned}
$$

with

$$
\boldsymbol{\delta}(\tau) \equiv\left\{\delta\left(\tau-\tau_{1}\right) \delta\left(\tau-\tau_{2}\right) \cdots \delta\left(\tau-\tau_{K}\right)\right\},
$$

where $\tau_{k}(k=1,2, \ldots, K)$ is the delay of the $k$ th delay path and $\delta$ is the delta function.

3.2.3. Weight Matrix for Delayed Waves. For a combination of input port $m$ and delayed wave $k$, we generate an uncorrelated Rayleigh variation between all of the delayed waves having different delays. The values of $L, M$, and $K$ are each expressed as a power of 2 , and it is assumed that $L=K M$ in principle: for example, $M=2, K=8$, and $L=16$, or $M=4, K=8$, and $L=32$. However, in the case of $L=32$, setting of smaller values for $M$ and $K$, such as $M=2$ or $K=6$, causes no serious problems by considering that 2 of 4 elements in $M=4$ and 2 of 8 elements in $K=8$ have a value of 0 . Here, the probe antenna input signal vector $\mathbf{v}_{k}$ for a delayed wave $k$ is given as

$$
\mathbf{v}_{k}(t)=\mathbf{w}_{\text {delay }}^{(k)} \otimes \mathbf{u}_{k}(t)
$$

with

$$
\mathbf{W}_{\text {delay }} \equiv\left(\begin{array}{lllll}
\mathbf{w}_{\text {delay }}^{(1)} & \cdots & \mathbf{w}_{\text {delay }}^{(k)} & \cdots & \mathbf{w}_{\text {delay }}^{(K)}
\end{array}\right),
$$

where $\otimes$ denotes the Kronecker product, which is used to construct a vector extended to the dimension of $K M$. The vector $\mathbf{w}_{\text {delay }}^{(k)}$ is the $k$ th element of the weight matrix $\mathbf{W}_{\text {delay }}(K \times K$ matrix $)$ composed of $K$ Walsh-Hadamard code vectors. Accordingly, the matrix of $\mathbf{W}_{\text {delay }} \otimes \mathbf{W}_{\mathrm{TX}}$ is dimensionally extended to $K M \times K M$.

The input signal $x_{l}$ for the respective probe antenna $l$ comprises a summation of all the delayed waves. Taking $\mathbf{x}$ to be the input vector for the probe antenna array having $x_{l}$ as its element, we then have

$$
\mathbf{x}(t)=\sum_{k=1}^{K} \operatorname{diag}\left(\mathbf{b}_{k}\right) \mathbf{v}_{k}(t)
$$

with

$$
\mathbf{B} \equiv\left(\begin{array}{lllll}
\mathbf{b}_{1} & \cdots & \mathbf{b}_{k} & \cdots & \mathbf{b}_{K}
\end{array}\right),
$$

where vector $\mathbf{b}_{k}$ represents the real-valued amplitudes in the angular direction of each probe antenna for the delayed wave $k$.

The proposed configuration is shown in Figure 6, and the overall channel model is represented by the following equation:

$$
\begin{aligned}
& \mathbf{H}_{\mathrm{FE}}(t, \tau)=\mathbf{A}_{\mathrm{RX}} \mathbf{A}_{\text {Doppler }}(t) \\
& \cdot \sum_{k=1}^{K} \operatorname{diag}\left(\mathbf{b}_{k}\right)\left\{\mathbf{w}_{\text {delay }}^{(k)} \otimes \mathbf{W}_{\mathrm{TX}}\right\} \delta\left(\tau-\tau_{k}\right) .
\end{aligned}
$$

3.3. TX Connection Matrix Incorporating TX-Side Spatial Correlation. For the angular profile of path directions in mobile communication systems, a Laplacian distribution with the center in the direction of the mobile terminal can be assumed at the base station side [36]. The angular profile $\Omega(\theta)$ and spatial correlation coefficient $\rho_{a}(\Delta x)$ can be written as follows:

$$
\begin{aligned}
\Omega(\theta) & =\frac{P_{R}}{\sqrt{2} \sigma_{\theta}} \exp \left(-\frac{\sqrt{2}\left|\theta-\theta_{0}\right|}{\sigma_{\theta}}\right), \\
\rho_{a}(\Delta x) & \approx \frac{1}{1+\left(\beta \Delta x \sigma_{\theta} \sin \theta_{0}\right)^{2} / 2} \exp \left(j \beta \Delta x \cos \theta_{0}\right),
\end{aligned}
$$

where $\beta$ is the wavenumber of the radio waves, $\theta$ is the arrival angle referenced to the baseline (direction of the array arrangement), $\theta_{0}$ is the central direction, and $\sigma_{\theta}$ is the standard deviation of the path direction angles. Equation (11) is an approximation conditioned on the angular spread not being overly large $\left[\sigma_{\theta} \ll 1(\mathrm{rad})\right]$ and is not valid when $\theta_{0}$ is approximately equal to 0 [37].

The spatial correlation matrix $\mathbf{R}_{\mathrm{TX}}$ between ports of the TX-side array antenna that comprises $M$ element antennas is represented by the following equation for a linear array equally spaced in intervals of $d_{t}$ in the direction of $\theta=0$ :

$$
\begin{aligned}
& \mathbf{R}_{\mathrm{TX}} \\
& =\left(\begin{array}{cccc}
1 & \rho_{a}\left(d_{t}\right) & \cdots & \rho_{a}\left\{(M-1) d_{t}\right\} \\
\rho_{a}^{*}\left(d_{t}\right) & 1 & \cdots & \rho_{a}\left\{(M-2) d_{t}\right\} \\
\vdots & \vdots & \ddots & \vdots \\
\rho_{a}^{*}\left\{(M-1) d_{t}\right\} & \rho_{a}^{*}\left\{(M-2) d_{t}\right\} & \cdots & 1
\end{array}\right) .
\end{aligned}
$$




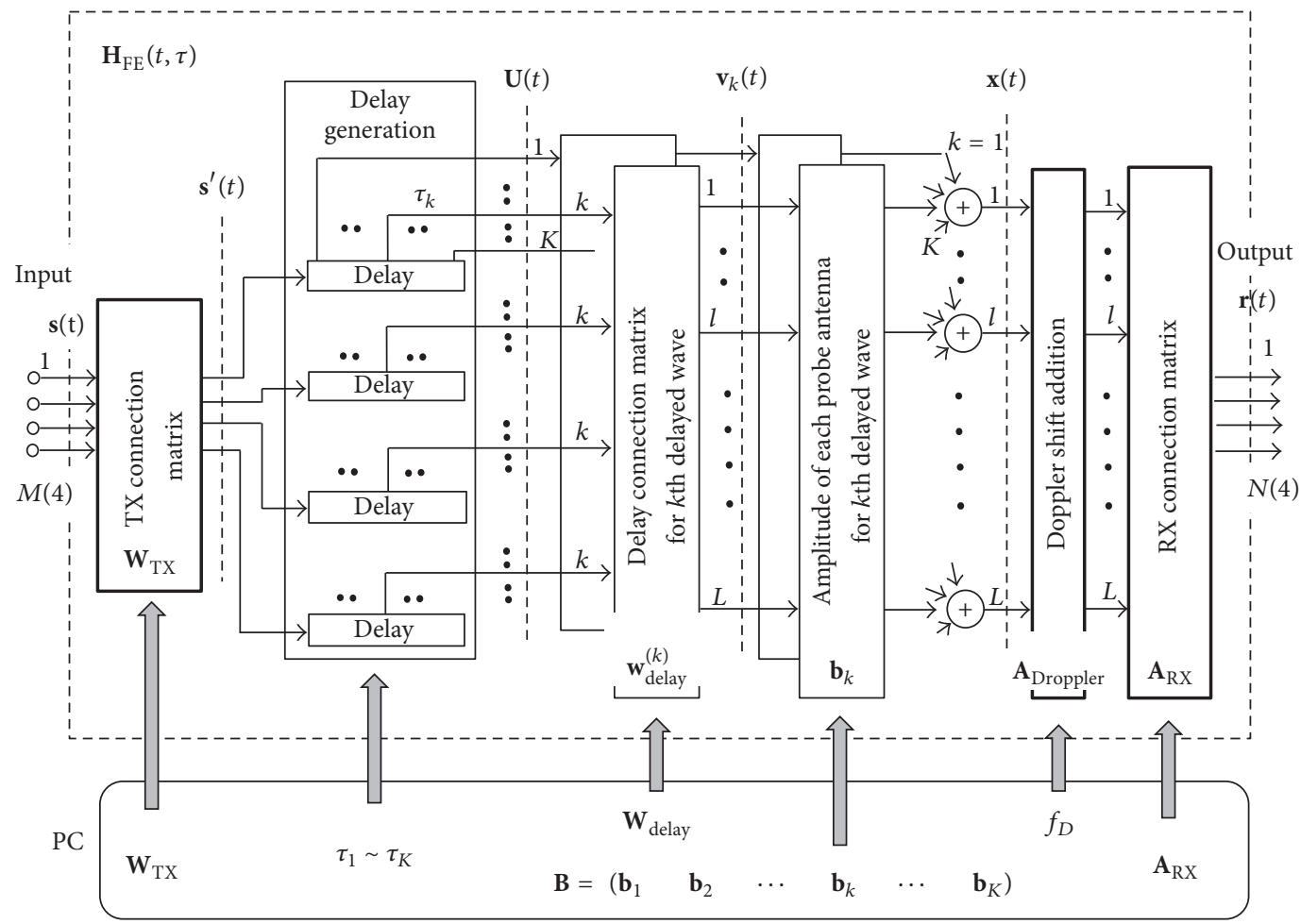

FIgURE 6: Overall configuration of the MIMO-FE proposed in this paper.

Owing to the eigenvalue properties for the above correlation matrix, the $M \times M$ connection matrix $\mathbf{W}_{\mathrm{TX}}$ that achieves spatial correlation for the TX-side is given by

$$
\begin{aligned}
& \mathbf{W}_{\mathrm{TX}}=\sqrt{M}\left(\mathbf{E}_{\mathrm{TX}} \mathbf{D}_{\mathrm{TX}}\right)^{\mathrm{T}}, \\
& \mathbf{D}_{\mathrm{TX}}=\operatorname{diag}\left(\begin{array}{llll}
\sqrt{\lambda_{\mathrm{TX} \_1}} & \sqrt{\lambda_{\mathrm{TX} \_2}} & \cdots & \sqrt{\lambda_{\mathrm{TX} \_M}}
\end{array}\right), \\
& \mathbf{E}_{\mathrm{TX}}=\left(\begin{array}{llll}
\mathbf{e}_{\mathrm{TX} \_1} & \mathbf{e}_{\mathrm{TX} \_2} & \cdots & \mathbf{e}_{\mathrm{TX} \_M}
\end{array}\right),
\end{aligned}
$$

where $\lambda_{\text {TX_m }}(m=1,2, \ldots, M)$ is the $m$ th eigenvalue of the correlation matrix $\mathbf{R}_{\mathrm{TX}}, \mathbf{e}_{\mathrm{TX} m}$ is the eigenvector corresponding to the eigenvalue $\lambda_{\mathrm{TX} \_m}$, and the superscript $T$ represents the transpose operation. Based on the scheme explained above, a function of the Kronecker model [16] for TX-side spatial correlation is implicitly incorporated.

To create independent fluctuations against all input signals, we recommend using the Walsh-Hadamard code matrix introduced in [11].

3.4. Computer Simulation for Channel Model Verification. A computer simulation was performed to verify the functionality of the proposed model represented by (9) and (13). First, the channel characteristics of the TX-side spatial correlation and the impact on telecommunications performance were examined in the case of a single delay wave for a narrowband Rayleigh fading environment. The specific settings were $M=N=4, K=1$ with amplitude vector $\mathbf{b}_{1}$ in (8b) for setting all 1 values, and $L=32$. If $\tau_{1}$ is set to 0 , then $\mathbf{H}_{\mathrm{FE}}$ in
(9) can be represented by the following equation using the narrow-band channel response matrix $\mathbf{A}_{\mathrm{FE}}$ :

$$
\mathbf{H}_{\mathrm{FE}}(t, \tau)=\mathbf{A}_{\mathrm{FE}}(t) \delta(\tau)
$$

The time-dependent correlation matrix $\mathbf{R}_{\mathrm{FE}}(t)$ of the channel is represented by

$$
\mathbf{R}_{\mathrm{FE}}(t)=\mathbf{A}_{\mathrm{FE}}(t) \mathbf{A}_{\mathrm{FE}}^{\mathrm{H}}(t),
$$

where superscript $\mathrm{H}$ represents the complex conjugate transpose.

The four eigenvalues of this channel correlation matrix are set as $\lambda_{1}(t)$ through $\lambda_{4}(t)$ in descending order. The element interval for both the TX-side and the RX-side arrays is set to one wavelength. Simulation results using the Kronecker model [16] are used as a reference to see whether the probability distribution of channel eigenvalues for the multipath environment generated by (14) is correctly performed.

The cumulative distributions of the four eigenvalues are shown in Figure 7, where the angular profile of the TX-side paths is a Laplacian distribution with $\theta_{0}=90^{\circ}$ and $\sigma_{\theta}=$ $5^{\circ}$. A case of no correlation between transmission ports, that is, the eigenvalues for an i.i.d. environment, is also shown. Comparison of channel capacities for $\theta_{0}=90^{\circ}, \sigma_{\theta}=$ $5^{\circ}$ and $\theta_{0}=30^{\circ}, \sigma_{\theta}=5^{\circ}$ are shown in Figure 8 for a signal-to-noise power ratio (SNR) of $10 \mathrm{~dB}$. As shown in Figures 7 and 8, the TX-side spatial correlation imposes large channel capacity degradations compared with the i.i.d. 


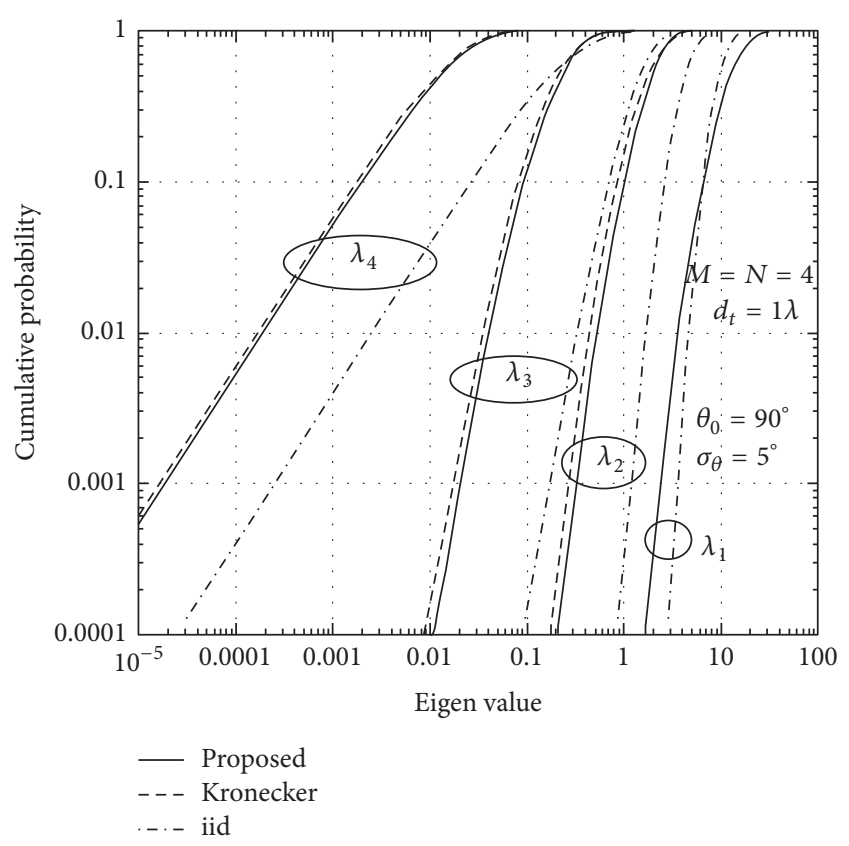

FIGURE 7: Eigenvalue characteristics when taking the TX-side spatial correlation into consideration.

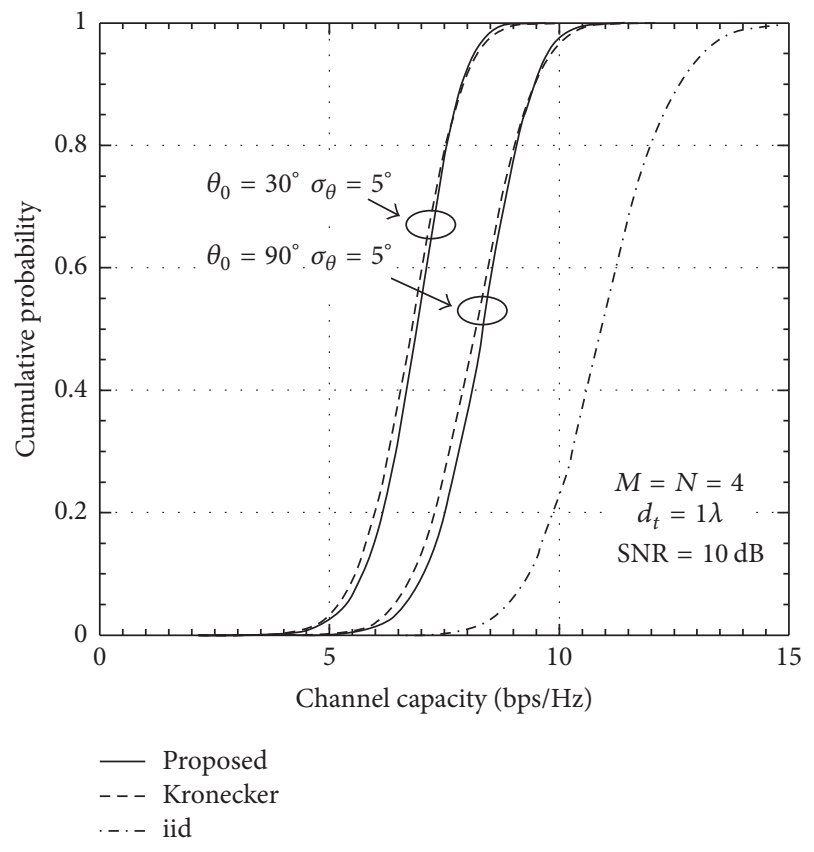

Figure 8: Comparison of channel capacities.

environment. The figures show that taking the TX-side spatial correlation into consideration is not a negligible matter. Moreover, the eigenvalue distribution and channel capacity distribution in the generated channel are matched extremely well to the simulation values of the Kronecker model. The appropriateness of the model in evaluating narrow-band transmission characteristics is thus verified.

Regarding the generation of wideband channel characteristics, parameters were set to $M=4, K=4, L=32$, and
$N=1$, and the delay wave amplitude vectors were set to $\mathbf{b}_{1}=(1,1, \ldots, 1), \mathbf{b}_{2}=0.8 \mathbf{b}_{1}, \mathbf{b}_{3}=0.6 \mathbf{b}_{1}$, and $\mathbf{b}_{4}=0.4 \mathbf{b}_{1}$. We examined the reception signal $r^{(m, n, k)}(t)$, which was set as the port $n$ output signal for delay wave $k$ from the port $m$ input signal. For this signal, we performed the following correlation operation without amplitude normalization:

$$
\gamma_{m m^{\prime}}^{\left(k k^{\prime}\right)}=\left\langle\left\{r^{(m, 1, k)}(t)\right\}^{*} r^{\left(m^{\prime}, 1, k^{\prime}\right)}(t)\right\rangle .
$$

An example of the expected values in the environment established with the above parameters, for $m=m^{\prime}$ and $k=k^{\prime}$, would be $1,0.64\left(0.8^{2}\right), 0.36\left(0.6^{2}\right)$, and $0.16\left(0.4^{2}\right)$ for $k=1,2$, 3 , and 4 , respectively, and for $k \neq k^{\prime}$ would be 0 .

In Table 1 , a comparison is made for $\left|\gamma_{m m^{\prime}}{ }^{(k, k)}\right|$ between these given settings (theoretically expected values) and values with generated signals (simulated values) as "generated/expected." The table shows that the values match up almost perfectly. Moreover, for conditions of $k \neq k^{\prime}$, where the value $\left|\gamma_{m m^{\prime}}{ }^{(k, k)}\right|$ should be 0 , all combinations were confirmed to be less than 0.001 . Thus, the delay wave environment was clearly generated as expected. From this validation, we can conclude that the multipath delay environment generated by (9) provides a wideband MIMO propagation environment.

\section{System Implementation on an FPGA}

In this section, we describe the FPGA implementation of the MIMO-FE incorporating the two-stage functions described in Section 3. Multipath fading for MIMO with any combination of $N \leq 4$ and $M \leq 4$ can be created. The FPGA used in this system is a Xilinx Virtex-6, LX240T. The prototype system specifications are given in Table 2. The program language is VHDL, and signals in the FPGA are created in parallel, as shown in Figure 6. The resource consumption ratios in the FPGA are about $90 \%$ for block RAM (RAMB36E1), which creates all delay paths, and less than $20 \%$ for multipliers (DSP48E1), which create the logic circuits. The interface connecting the FPGA and a personal computer (PC) is USB 2.0, and its protocol is UART (universal asynchronous receiver transmitter). Fading emulation can be carried out in real-time operation, while the parameter setting is done offline before starting the emulation.

The operation clock is synchronized at $160 \mathrm{MHz}$ with the input, output, and signal-processing units. The input and output signals are specified by an intermediate frequency (IF) band of $f_{\mathrm{IF}}=40 \mathrm{MHz}$, a signal bandwidth of $W=$ $40 \mathrm{MHz}$, and an accordingly fractional bandwidth $W / f_{\mathrm{IF}}$ of 1. For distortion-free signal transmission at this bandwidth, we use the digital filter configuration shown in Figure 9(a), in which the Hilbert transformation output $y^{(Q)}$ exhibits pass characteristics, as shown in Figure 9(b), allowing the $40-\mathrm{MHz}$-band signal to be transmitted without distortion. When calculating complex weighting $w\left(w^{(I)}+j w^{(\mathrm{Q})}\right)$ to the input signal $x$, the real-value output $z$ can be obtained from $z=w^{(I)} y^{(I)}-w^{(\mathrm{Q})} y^{(\mathrm{Q})}$, where $y^{(I)}$ and $y^{(\mathrm{Q})}$ are given in Figure 9(a). In this way, all real-value signal processing on the IF stage can be accomplished in the FPGA. 


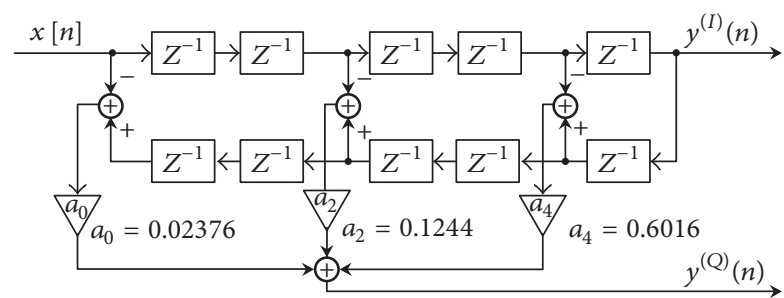

(a) Circuit configuration

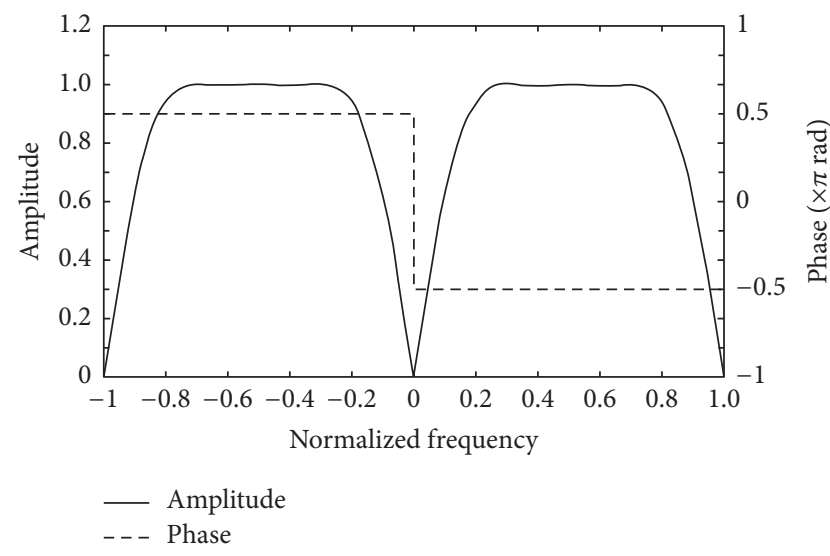

(b) Amplitude and phase characteristics

Figure 9: Hilbert transformation circuit for realizing a relative bandwidth equal to 1.

TABLE 1: Delayed path correlation in terms of $|\gamma|$ (the values in the table are "generated/expected").

\begin{tabular}{|c|c|c|c|c|c|}
\hline$k=k^{\prime}$ & $m \backslash m^{\prime}$ & 1 & 2 & 3 & 4 \\
\hline \multirow{4}{*}{1} & 1 & $0.9975 / 1.0000$ & $0.8680 / 0.8693$ & $0.6228 / 0.6245$ & $0.4245 / 0.4250$ \\
\hline & 2 & & $0.9996 / 1.0000$ & $0.8684 / 0.8693$ & $0.6247 / 0.6245$ \\
\hline & 3 & & & $0.9987 / 1.000$ & $0.8692 / 0.8693$ \\
\hline & 4 & & & & $0.9999 / 1.0000$ \\
\hline \multirow{4}{*}{2} & 1 & $0.6396 / 0.6400$ & $0.5555 / 0.5564$ & $0.3994 / 0.3977$ & $0.2715 / 0.272$ \\
\hline & 2 & & $0.6385 / 0.6400$ & $0.5557 / 0.5564$ & $0.3989 / 0.3977$ \\
\hline & 3 & & & $0.6402 / 0.6400$ & $0.5561 / 0.5564$ \\
\hline & 4 & & & & $0.6394 / 0.6400$ \\
\hline \multirow{4}{*}{3} & 1 & $0.3602 / 0.3600$ & $0.3129 / 0.3130$ & $0.2251 / 0.2248$ & $0.1532 / 0.1530$ \\
\hline & 2 & & $0.3597 / 0.3600$ & $0.3132 / 0.3130$ & $0.2249 / 0.2248$ \\
\hline & 3 & & & $0.3607 / 0.3600$ & $0.3134 / 0.3130$ \\
\hline & 4 & & & & $0.3602 / 0.3600$ \\
\hline \multirow{4}{*}{4} & 1 & $0.1598 / 0.1600$ & $0.1390 / 0.1391$ & $0.0998 / 0.0999$ & $0.0681 / 0.0681$ \\
\hline & 2 & & $0.1601 / 0.1600$ & $0.1391 / 0.1391$ & $0.1001 / 0.0999$ \\
\hline & 3 & & & $0.1600 / 0.1600$ & $0.1393 / 0.1391$ \\
\hline & 4 & & & & $0.1603 / 0.1600$ \\
\hline
\end{tabular}

Figure 10 shows an external view of the FE as implemented on the FPGA for all functions shown in Figure 6. The overall system is highly compact, as shown here by its size when mounted in a case measuring $28 \times 22 \times 5 \mathrm{~cm}$. A functional schematic of the created multipath environment in the FPGA is given in Figure 4(b) for $L=32, K=8$, and $M=N=4$.

For functional verification, we first set the parameters to their most simple terms, namely, $M=1, K=1$, and $N=1$, and we inputted an unmodulated signal into input port 1. Figure 11 shows the power spectrum of port 1 output at $f_{D}$ $=200 \mathrm{~Hz}$ and $\mathbf{b}_{1}=\mathbf{1}$ when received by an omnidirectional antenna (see Figure 13, Case I). As discussed in [11], in order to exclude the same Doppler shift when setting the probe antennas symmetrically, we adopted small angular offsets to each probe antenna direction. Through the addition of a

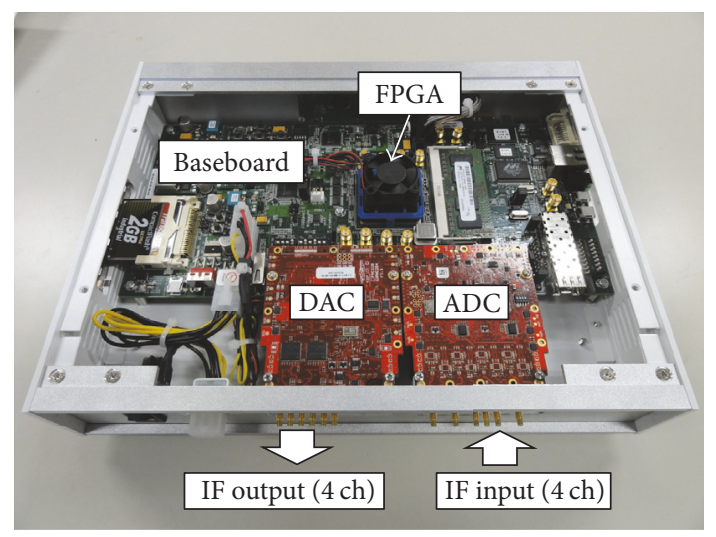

Figure 10: MIMO-FE as implemented on an FPGA (case size is 28 $\times 22 \times 5 \mathrm{~cm})$. 
TABLE 2: MIMO FE system specifications.

\begin{tabular}{lc}
\hline FPGA IC & $\begin{array}{c}\text { XILINX Virtex 6 LX240T } \\
\text { Baseboard }\end{array}$ \\
\hline Input/output & \\
ADC & 4DSP FMC104 (14 bit) \\
DAC & 4DSP FMC204 (16 bit) \\
Input ports $M$ & 4 \\
Output ports $N$ & 4 \\
\hline Signal processing & \\
Clock frequency $f_{s}$ & $160 \mathrm{MHz}$ \\
IF frequency & $40 \mathrm{MHz}$ \\
Bandwidth & $40 \mathrm{MHz}(\mathrm{max})$ \\
\hline Propagation parameters & 32 \\
Probe antennas $L$ & 8 \\
Delay paths $K$ & $50 \mu \mathrm{s}(k=1-6), 200 \mu \mathrm{s}(k=7,8)$ \\
Maximum delay & $6.25 \mathrm{~ns}\left(f_{s}=160 \mathrm{MHz}\right)$ \\
Delay resolution & $0.1 \mathrm{~Hz} \sim 10 \mathrm{kHz}$ \\
Doppler frequency &
\end{tabular}

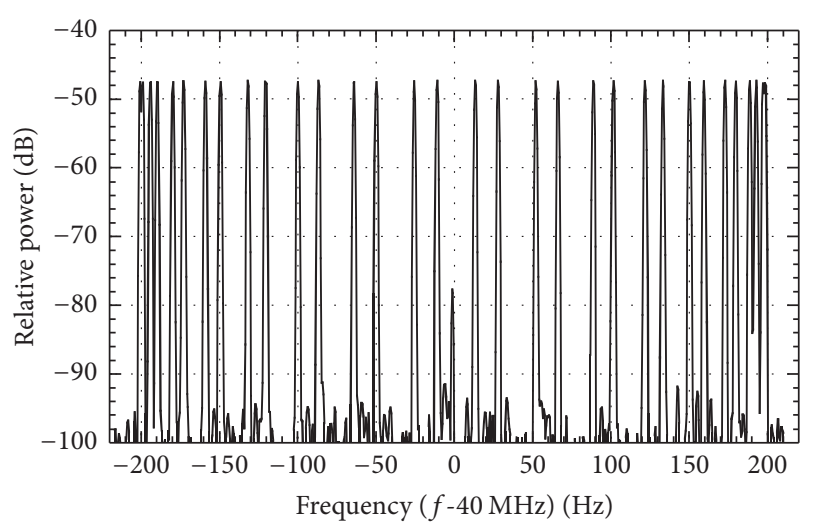

FIGURE 11: Doppler spectrum for $M=N=K=1$ with $f_{D}=200 \mathrm{~Hz}$ received by an omnidirectional antenna.

small angular offset in (3), 32 Doppler shifts corresponding to each probe antenna position are clearly seen in the figure. Although the average power radiating from each probe antenna is uniform, the averages' spectral density profile becomes approximately U-shaped because the spectral density in the frequency axis becomes higher towards the frequency of $\pm f_{D}$. This condition corresponds to the Jakes model. Moreover, the system has a function to realize various channel models having an arbitrary angular power profile by adjusting the amplitude of radiated signals from the probe antennas [factors of $\mathbf{b}$ in (8b)].

With the four receiving antennas $(N=4)$ in a cardioid pattern, we rotated the beam direction in increments of $90^{\circ}$ for each antenna and entered the characteristics into the receiving connection matrix $\mathbf{A}_{\mathrm{RX}}$ (see Figure 13, Case III). Figure 12 shows the received signal spectra of two output ports ( $n=1$ and $n=2)$. The figure indicates, as shown by the shape of the cardioid pattern, that each of the antennas exhibited strong reception for as many as 16 waves in the beam direction and that the spectra corresponded to antenna direction (the directivity rotation direction).

The generated channel characteristics for input port $m$, output port $n$, and delayed wave $k$ are denoted by $a_{n m}{ }^{(k)}(t)$. We statistically tested amplitude distribution, eigenvalue characteristics, and correlation characteristics and confirmed them as expected. We also found that, for all combinations of delayed waves, $a_{n m}{ }^{(k)}$ and $a_{n m^{\prime}}{ }^{\left({ }^{\prime}\right)}$ were perfectly uncorrelated except for $k=k^{\prime}$.

Regarding the combination of $N$ and $M$, there is no particular restriction from the system performance viewpoint in principle. For example, when $M=1$ and $N=4$, the effectiveness is demonstrated in the evaluation of maximalratio combining (MRC) diversity (see Section 5.1). Moreover, as shown in Table 1, performance is also good when $M=4$ and $N=1$. Therefore, an imbalance between $M$ and $N$ does not limit the performance of the circuit configuration. However, in FPGA implementation, performance is limited by the gate array resources in the circuit. As the values of $N$ and $M$ increase, for example, in systems such as massive MIMO, the number of probe antennas $L$ must also be increased in order to realize accurate eigenvalue characteristics. With such a scale, in commercially available FPGAs, the number of gates in the circuit becomes insufficient. Therefore, the scale size with arbitrary combination of $M=N=4$ or less is a practical limit to satisfy the specifications in this prototype.

\section{Application to Wireless System Performance Evaluations}

In this section, we will demonstrate the effectiveness of our MIMO-FE system with two application examples.

5.1. Digital Broadcasting Signal Reception with MRC Diversity in Mobile Environments. Terrestrial digital broadcasting systems in Japan operate in the ultra-high-frequency band and conform to the Integrated Services Digital Broadcasting-Terrestrial (ISDB-T) standard (bandwidth of $5.6 \mathrm{MHz}$, orthogonal frequency-division multiplexing [OFDM] modulation using 5,617 subcarriers). In this study, we assessed the reception characteristics of a television receiver (Panasonic TH-L17F1) equipped with a commercial four-antenna diversity function with MRC on an individual subcarrier base. Specifically, we generated two types of multipath environments, a "fast fading" environment, and a "large delay difference" environment using the FE and compared a single antenna and four-antenna array.

We assessed diversity reception performance for the following four cases.

Case I. Single omnidirectional antenna is placed $(N=1)$.

Case II. Four omnidirectional antennas are placed one wavelength from the center in a square array $(N=4)$.

Case III. Four cardioid-shape directional antennas are placed as in Case II with beams radiating in directions opposed to the center (see Figure 12). 

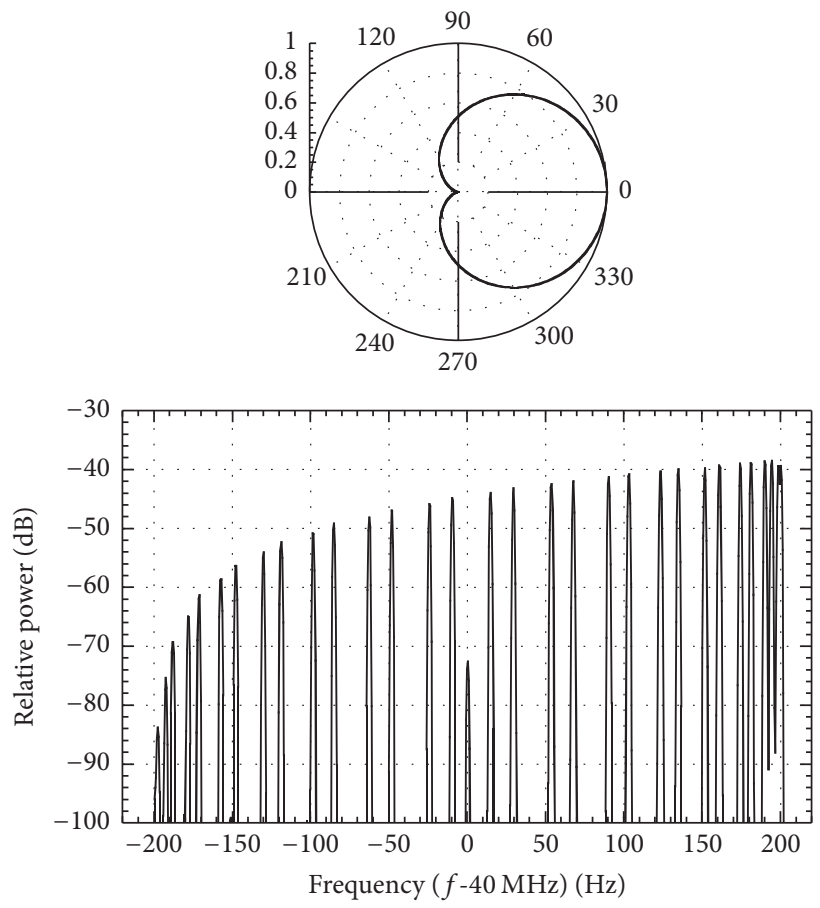

(a) $n=1$
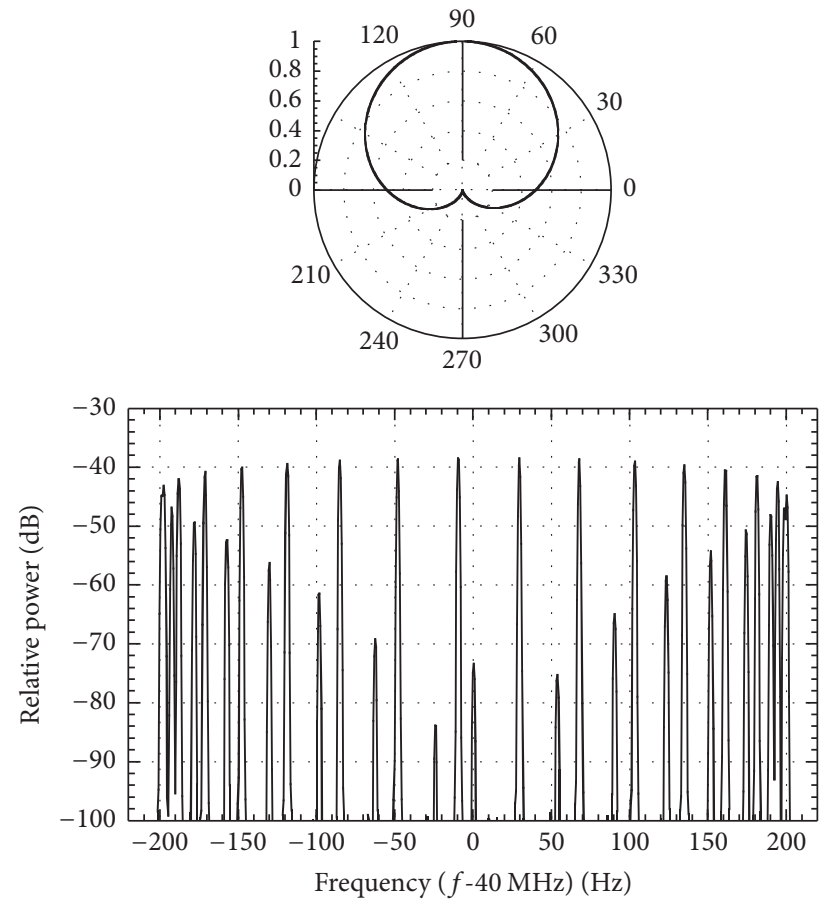

(b) $n=2$

FIGURE 12: Receiving antenna directivity patterns and concurrent Doppler spectra.

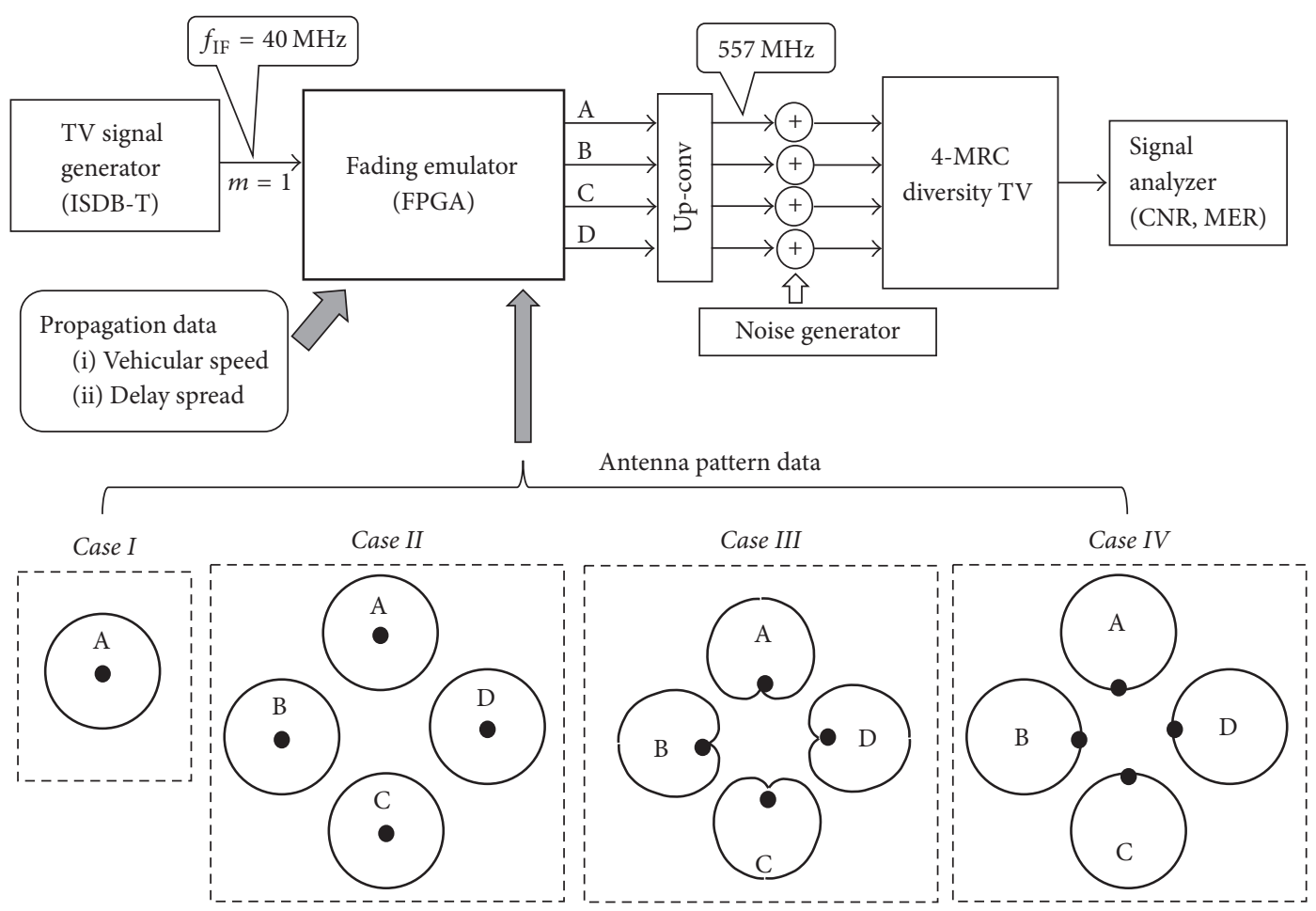

FIGURE 13: Experiment configuration for ISDB-T diversity characteristic assessment. 


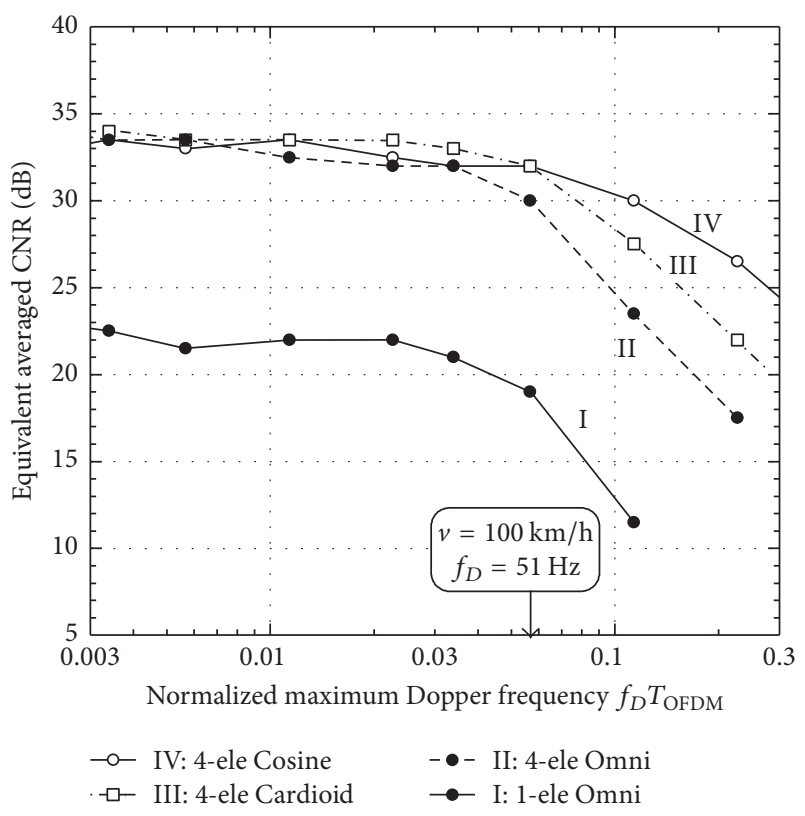

FIgURE 14: Fast fading tolerance for TV signal MRC reception performance in various antenna configurations.

Case IV. Four cosine-shape (half-side only) directional antennas are placed as in Case III.

Considering the omnidirectional antenna pattern of $g_{\mathrm{I}}(\theta)=g_{\mathrm{II}}(\theta)=1$ for the whole angular range in Cases I and II, we set $g_{\mathrm{III}}$ for Case III to $g_{\mathrm{III}}(\theta)=a_{\mathrm{III}}(1+\cos (\theta-(n-1) \pi / 2))$ and $g_{\mathrm{IV}}$ for Case IV at $g_{\mathrm{IV}}(\theta)=a_{\mathrm{IV}} \cos (\theta-(n-1) \pi / 2)$, where $a_{\mathrm{III}}=\sqrt{2 / 3}$, while $a_{\mathrm{IV}}=2$ for $|\theta| \leq \pi / 2$ and $a_{\mathrm{IV}}=0$ for $|\theta|>\pi / 2$. The coefficient $a$ is the gain-adjustment factor so that the electric power integral in the horizontal plane is the same as for the omnidirectional antenna.

Figure 13 shows the measurement system with the antenna positioning described above. An ISDB-T signal with a center frequency of $40 \mathrm{MHz}$ is input to a port $(m=1)$ and the output of each antenna was obtained with $n=1$ for Case I and $n=1,2,3$, and 4 for Cases II, III, and IV. The outputs of each were up-converted from $40 \mathrm{MHz}$ to $557 \mathrm{MHz}$ to convert to the television frequency band. The up-converted signals were connected directly to the ports of the television receiver after disconnecting the existing antennas, and a noise signal was added to the input to adjust the carrier-to-noise power ratio (CNR). For MRC evaluation performance in fast fading conditions, we set $K=3$ with $\mathbf{b}_{1}=\mathbf{b}_{2}=\mathbf{b}_{3}=(1,1, \ldots, 1), \tau_{1}$ $=0, \tau_{2}=1 \mu \mathrm{s}$, and $\tau_{2}=2 \mu \mathrm{s}$. Under these conditions, reception was investigated while the maximum Doppler frequency was changed.

Figure 14 shows the equivalent average CNR values relative to the normalized maximum Doppler frequency $f_{D} T_{\text {OFDM }}$, where $T_{\text {OFDM }}$ of $1.134 \mathrm{~ms}$ is the symbol period, including the guard interval (GI) in the OFDM signal. The results in this figure demonstrate that MRC diversity applications (Cases II, III, and IV) show a large improvement in characteristics. They also show that an array of higher

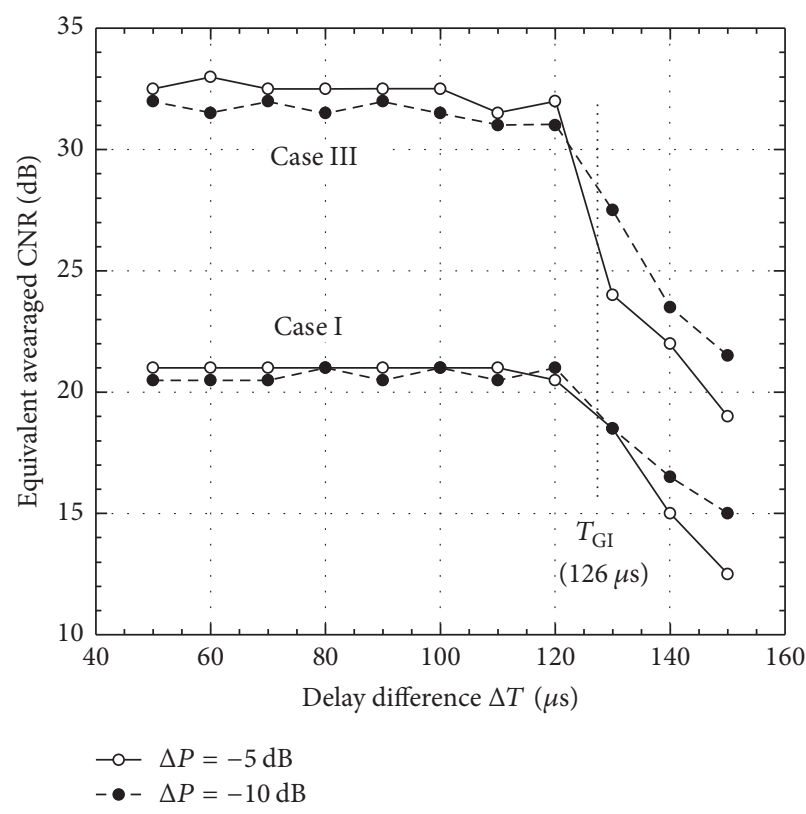

FIGURE 15: Delay difference tolerance for TV signal MRC reception performance in Cases I and III.

directional antennas (IV > III > II) has better tolerance against Doppler spreading in a multipath environment.

To demonstrate a related application, we evaluated the differential delay tolerance of MRC reception for ISDB-T signals. We set $K=4$, where $\mathbf{b}_{1}=\mathbf{b}_{2}=(1,1, \ldots, 1)$ and $\mathbf{b}_{3}=$ $\mathbf{b}_{4}=0.562 \mathbf{b}_{1}(\Delta P=-5 \mathrm{~dB})$ or $0.316 \mathbf{b}_{1}(\Delta P=-10 \mathrm{~dB})$ with $\tau_{1}=0, \tau_{2}=1 \mu \mathrm{s}, \tau_{3}=\Delta T$, and $\tau_{4}=\Delta T+1 \mu \mathrm{s}$. Under these conditions, the delay difference $\Delta T$ is varied. Figure 15 shows the equivalent average CNR values as a function of delay difference $\Delta T$ for Cases I and III. Considering that the GI period is $126 \mu \mathrm{s}$ and that the apparent degradation can be seen for $\Delta T$ exceeding the GI period, the results obtained are reasonable.

5.2. Wireless Local Area Networks. Evaluation of transmission characteristics is a relatively simple matter in cases of oneway transmission, as described above. In cases where a transmission signal is controlled by reception acknowledgments (ACK/NAK), the measurement procedure is more complex because of the need to set up a feedback loop. The following demonstration provides an example assessment procedure for an IEEE 802.11n-compliant WLAN.

The WLAN base unit (i.e., the access point, AP) used in this experiment was a Buffalo WZR-AMPG300NH router, designed to IEEE $802.11 \mathrm{a} / \mathrm{b} / \mathrm{g} / \mathrm{n}$ specifications, with two antenna ports for transmission and reception and one for reception only. In our measurement, the 802.11n mode with $40-\mathrm{MHz}$ bandwidth in the $5-\mathrm{GHz}$ band is used for data transmission. Its theoretical maximum speed is $300 \mathrm{Mbps}$. In this experiment, the original-equipment antennas were replaced with directly connected signal cables. The user equipment (UE) used in this experiment was an Intel Centrino Advanced-N 6200 adapter, which has the same specifications as the AP unit. It was mounted on a board to 


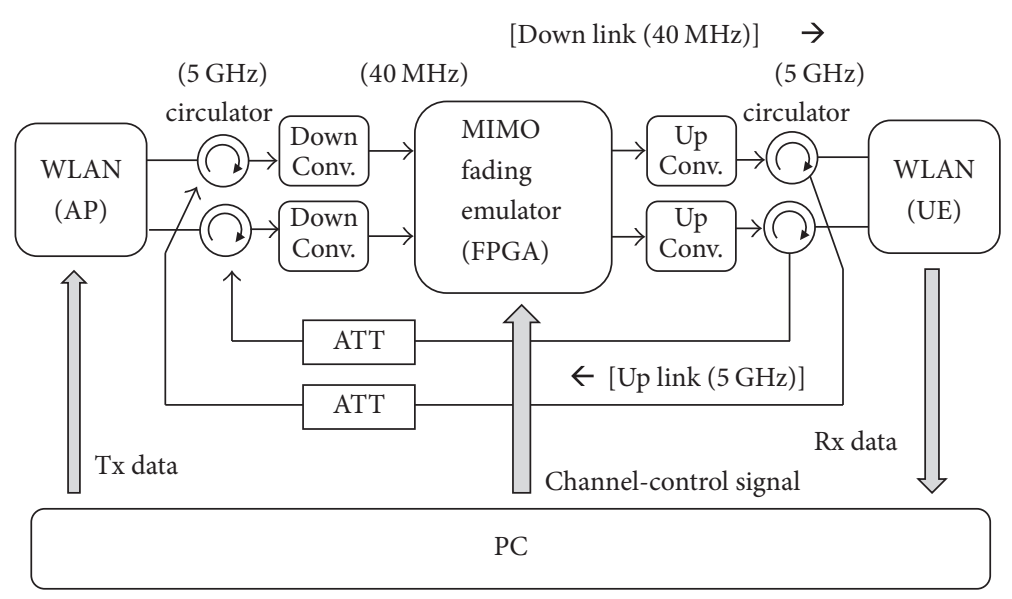

FIGURE 16: Experiment configuration for WLAN transmission characteristics assessment.

convert mini PCI Express to PCI Express and then installed on a desktop PC. For the throughput measurement, we used two software programs we wrote. One program is for transmitting very large amounts of data from the AP to the $\mathrm{UE}$, and the other is for measuring throughput performance at the UE. Both software programs are installed on the PC. Preliminary measurement of the throughput was about $100 \mathrm{Mbps}$ with direct cable connection and appropriate level adjustment but without down- and up-conversion.

The WLAN evaluation experiment configuration, as shown schematically in Figure 16, included $2 \times 2$ MIMO communication channels with fading added by the emulator to the downlink ( $\mathrm{AP} \rightarrow \mathrm{UE}$ ). Since the fading addition was performed in the $40-\mathrm{MHz}$ band, down- and up-converters were introduced before and after the emulator, respectively. For upand down-converters, we made circuits using commercially available radiofrequency components, such as mixers, lowpass filters, and circulators with the circuit configuration in Figure 16. The uplink (AP $\leftarrow \mathrm{UE}$ ) was for ACK/NAK signal transmission only and was therefore directly connected by a cable without frequency conversion. The transmission and reception signals were separated by a circulator, and unwanted signals were suppressed using attenuators to avoid leakage of the downlink signal via the uplink channel. The transmission signal from the $\mathrm{PC}$ was continuously sent to the PC receiving end without interruption, and the throughput was assessed with our evaluation software.

Figure 17 shows an example of this evaluation where the throughput in terms of bits per second is depicted as a function of the maximum Doppler frequency and delay difference between two equally powered delayed waves. Only a relative trend for the throughput behavior against the delay and Doppler spread can be presented here, since there is no reference channel for making an absolute comparison. As can be seen from the figure, in Rayleigh fading environments, statistically stable characteristics are obtained within a range of $f_{D}=100 \mathrm{~Hz}$ and a delay difference of about $400 \mathrm{~ns}$. For IEEE $802.11 \mathrm{n}, \mathrm{GI}=800 \mathrm{~ns}$ is the standard, but when the delay spread is smaller, there is also a mode that operates with GI $=400 \mathrm{~ns}$. Therefore, in the vicinity of the delay of $400 \mathrm{~ns}$, the operation

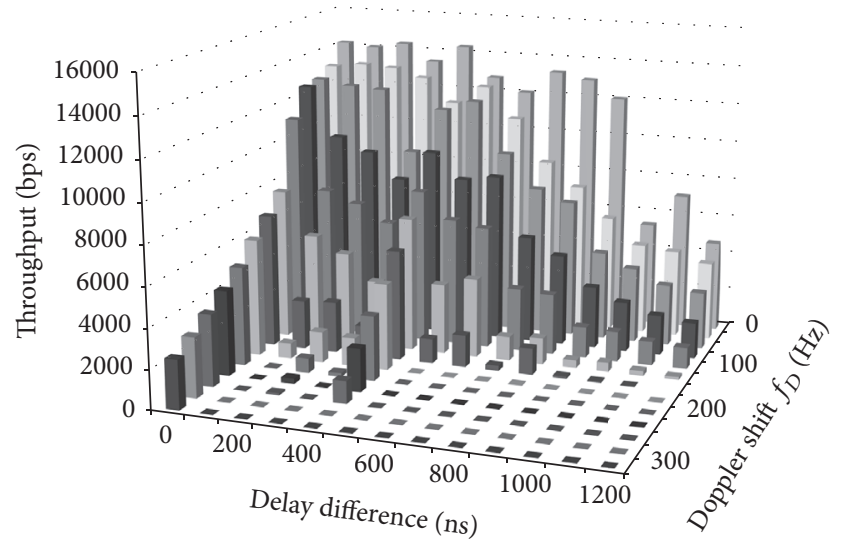

FIGURE 17: Results of WLAN throughput as a function of Doppler spread and delay spread.

tends to become unstable because it is in the threshold position of this switching. A Doppler shift of $f_{D}=100 \mathrm{~Hz}$ corresponds to the normalized maximum Doppler frequency $f_{D} T_{\text {OFDM }}\left(T_{\text {OFDM }}=4 \mu \mathrm{s}\right)$ of approximately 0.0004 , which never falls into the category of fast fading but is degraded nonetheless. This may be attributed to the fact that WLAN is designed under the assumption of slow-moving indoor conditions; that is, fast fading tolerance is not considered as a design objective. No further details concerning the WLAN performance are given here, since the objective of the evaluation was to demonstrate the effectiveness of the emulator and not to assess commercial WLAN.

\section{Conclusions}

In this study, we achieved implementation of a two-stage MIMO-FE circuit on an FPGA with an extremely simple configuration that is able to generate a variety of fading environments. The input of propagation parameters and receiving antenna characteristics from a PC allows it to 
produce a $4 \times 4$ MIMO fading environment in real-time. The results are summarized as follows.

(i) We introduced a more simplified configuration of a multipath fading generation unit, that is, the "advanced antenna-branch-controlled" method. The scheme can drastically reduce the number of delay units in the $L \times M$ connection matrix, which achieves $L=32$ probe antennas with 8 delay paths varying up to $200 \mu \mathrm{s}$.

(ii) In order to realize the propagation characteristics around the base station site, the TX-side spatial correlation property was incorporated, and its validity was confirmed through computer simulation.

(iii) We successfully demonstrated two evaluations of transmission characteristics: application to WLAN and terrestrial digital broadcasting diversity reception.

The next goal is to apply our method to the performance evaluation of next-generation wireless terminals.

\section{Conflicts of Interest}

The authors declare that there are no conflicts of interest regarding the publication of this paper.

\section{References}

[1] H. Arai, Measurement Science and Technology, Artech House, 2001.

[2] GPP, "Verification of radiated multi-antenna reception performance of user equipment (UE)," Tech. Rep., 2015, Tech. Rep. (TR37.977 v13.1.0), 2015.

[3] H. Iwai, K. Sakaguchi, T. Sakata, and A. Yamamoto, "Proposal of spatial fading emulator dedicated for performance evaluation of handset antennas," IEICE Transactions on Communications, vol. J91-B, no. 9, pp. 960-971, 2008.

[4] T. Sakata, A. Yamamoto, K. Ogawa, and J.-I. Takada, "MIMO channel capacity measurement in the presence of spatial clusters using a fading emulator," in Proceedings of the 2009 IEEE 20th Personal, Indoor and Mobile Radio Communications Symposium, PIMRC 2009, pp. 97-101, September 2009.

[5] P. Kyösti, J.-P. Nuutinen, and T. Jämsä, "MIMO OTA test concept with experimental and simulated verification," in Proceedings of the 4th European Conference on Antennas and Propagation, EuCAP 2010, pp. 1-5, April 2010.

[6] P. Hallbjörner, Z. Ying, M. Håkansson, C. Wingqvist, T. Anttila, and J. Welinder, "Multipath simulator for mobile terminal antenna characterisation," IET Microwaves, Antennas and Propagation, vol. 4, no. 2, pp. 743-750, 2010.

[7] W. Fan, C. B. Lisbona, F. Sun, J. O. Nielsen, M. B. Knudsen, and G. F. Pedersen, "Emulating spatial characteristics of MIMO channels for OTA testing," IEEE Transactions on Antennas and Propagation, vol. 61, no. 8, pp. 4306-4314, 2013.

[8] R. Mehmood, M. A. Jensen, and J. W. Wallace, "Reconfigurable OTA chamber: A new paradigm for testing of MIMO wireless devices," in Proceedings of the 6th IEEE International Symposium on Microwave, Antenna, Propagation, and EMC Technologies, MAPE 2015, pp. 591-594, October 2015.
[9] A. Khatun, V.-M. Kolmonen, V. Hovinen et al., "Experimental verification of a plane-wave field synthesis technique for MIMO OTA antenna testing," IEEE Transactions on Antennas and Propagation, vol. 64, no. 7, pp. 3141-3150, 2016.

[10] A. Kosako, M. Shinozawa, and Y. Karasawa, "A simplified configuration of fading emulator system for MIMO-OTA testing," IEICE Transactions on Communications, vol. J95-B, no. 2, pp. 275-284, 2012.

[11] S. Pasisingi, K. Nakada, A. Kosako, and Y. Karasawa, "Performance evaluation of a MIMO channel model for simplified OTA test systems," Eurasip Journal on Wireless Communications and Networking, vol. 2013, no. 1, article 285, 2013.

[12] Y. Karasawa., Y. Gunawan, S. Pasisingi, K. Nakada, and A. Kosako, "Development of a MIMO-OTA system with simplified configuration," Journal of the Korean Institute of Electromagnetic Engineering and Scinece, vol. 12, no. 1, pp. 77-84, 2012.

[13] K. Nakada and Y. Karasawa, "FPGA implementation and evaluation of a simplified MIMO-OTA system," IEICE Transactions on Communications, vol. J97-B, no. 1, pp. 51-60, 2014.

[14] Y. Jing, Z. Wen, H. Kong, S. Duffy, and M. Rumney, “Two-stage over the air (OTA) test method for MIMO device performance evaluation," in Proceedings of the 2011 IEEE International Symposium on Antennas and Propagation and USNC/URSI National Radio Science Meeting, APSURSI 2011, pp. 71-74, Washington, DC, USA, June 2011.

[15] G. Sun, K. Nakada, I. Oshima, and Y. Karasawa, "Simplified two-stage MIMO-OTA simulation scheme and its application to evaluation of antenna coupling effect," IEICE Transactions on Communications, vol. J96-B, no. 7, pp. 701-711, 2013.

[16] J. P. Kermoal, L. Schumacher, K. I. Pedersen, P. E. Mogensen, and F. Frederiksen, "A stochastic MIMO radio channel model with experimental validation," IEEE Journal on Selected Areas in Communications, vol. 20, no. 6, pp. 1211-1226, 2002.

[17] A. F. Molisch, "A generic model for MIMO wireless propagation channels in macro- and microcells," IEEE Transactions on Signal Processing, vol. 52, no. 1, pp. 61-71, 2004.

[18] M. A. Jensen and J. W. Wallace, "A review of antennas and propagation for MIMO wireless communications," IEEE Transactions on Antennas and Propagation, vol. 52, no. 11, pp. 28102824, 2004.

[19] W. Weichselberger, M. Herdin, H. Özcelik, and E. Bonek, "A stochastic MIMO channel model with joint correlation of both link ends," IEEE Transactions on Wireless Communications, vol. 5, no. 1, pp. 90-99, 2006.

[20] C. Xiao, J. Wu, S.-Y. Leong, Y. R. Zheng, and K. B. Letaief, "A discrete-time model for triply selective MIMO rayleigh fading channels," IEEE Transactions on Wireless Communications, vol. 3, no. 5, pp. 1678-1688, 2004.

[21] D. Kurita, Y. Okano, S. Nakamatsu, and T. Okada, "Experimental comparison of MIMO OTA testing methodologies," in Proceedings of the Fourth European Conference on Antennas \& Propagat (EuCAP 2010), pp. 1-5, April 2010.

[22] P.-S. Kildal, X. Chen, C. Orlenius, M. Franzen, and C. S. Patane, "Characterization of reverberation chambers for OTA measurements of wireless devices: physical formulations of channel matrix and new uncertainty formula," IEEE Transactions on Antennas and Propagation, vol. 60, no. 8, pp. 3875-3891, 2012.

[23] Y. Karasawa, I. Oshima, F. Tamrin, Y. Sakamoto, R. Arif, and K. Sasaki, "Fundamental propagation characteristics of stirrer-less reverberation chamber for MIMO-OTA measurements," IEICE Transactions on Communications, vol. E97B, no. 10, pp. 20832092, 2014. 
[24] J. Carlsson, C. P. Lötbäck, and A. A. Glazunov, "Reverberation chamber for OTA measurements: the history of a dream!", in Proceedings of the 2017 11th European Conference on Antennas and Propagation (EUCAP), pp. 237-241, Paris, France, March 2017.

[25] T. Laitinen, P. Kyösti, T. Jämsä, and P. Vainikainen, "Generation of a field with a Laplacian-distributed power azimuth spectrum scattered by a single cluster in a MIMO-OTA test system based on multiple probe antennas," in Proceedings of the 2010 Asia-Pacific Microwave Conference, APMC 2010, pp. 2127-2130, December 2010.

[26] P. Kyösti and L. Hentilä, "Criteria for physical dimensions of MIMO OTA multi-probe test setup," in Proceedings of the 6th European Conference on Antennas and Propagation, EuCAP 2011, pp. 2055-2059, 2011.

[27] L. J. Foged, A. Scannavini, N. Gross, and J. Estrada, "MIMO OTA testing using a multiprobe system approach," in Proceedings of the 2013 7th European Conference on Antennas and Propagation, EuCAP 2013, pp. 1673-1677, April 2013.

[28] W. Fan, J. Ø. Nielsen, O. Franek et al., "Antenna pattern impact on MIMO OTA testing," IEEE Transactions on Antennas and Propagation, vol. 61, no. 11, pp. 5714-5723, 2013.

[29] M. G. Nilsson, P. Hallbjörner, N. Arabäck, B. Bergqvist, T. Abbas, and F. Tufvesson, "Measurement uncertainty, channel simulation, and disturbance characterization of an over-theair multiprobe setup for cars at $5.9 \mathrm{GHz}$," IEEE Transactions on Industrial Electronics, vol. 62, no. 12, pp. 7859-7869, 2015.

[30] C. Wright and S. Basuki, "Utilizing a channel emulator with a reverberation chamber to create the optimal MIMO OTA test methodology," in Proceedings of the 2010 Global Mobile Congress, GMC'2010, pp. 1-5, October 2010.

[31] W. Yu, Y. Qi, K. Liu, Y. Xu, and J. Fan, "Radiated two-stage method for LTE MIMO use requipment performance evaluation," IEEE Transactions on Electromagnetic Compatibility, vol. 56, no. 6, pp. 1691-1696, 2014.

[32] F. Ren and Y. R. Zheng, "A novel emulator for discrete-time MIMO triply selective fading channels," IEEE Transactions on Circuits and Systems, vol. 57, no. 9, pp. 2542-2551, 2010.

[33] E. Johnson and R. Verret, "Advancing RF test with open FPGAs," in Proceedings of the 48th IEEE Systems Readiness Technology Conference, AUTOTESTCON 2012, pp. 126-129, September 2012.

[34] T. H. Loh, C. Li, H. Wang, and F. Qin, "A software-definedradio platform for multiple-input-multiple-output over-the-air measurement," in Proceedings of the 10th European Conference on Antennas and Propagation, EuCAP 2016, pp. 1-4, April 2016.

[35] P. Almers, E. Bonek, A. Burr et al., "Survey of channel and radio propagation models for wireless systems," EURASIP Journal on Wireless Communications and Networking, vol. 2007, no. 19070, pp. 1-19, 2007.

[36] K. I. Pedersen, P. E. Mogensen, and B. H. Fleury, "A stochastic model of the temporal and azimuthal dispersion seen at the base station in outdoor propagation environments," IEEE Transactions on Vehicular Technology, vol. 49, no. 2, pp. 437-447, 2000.

[37] Y. Karasawa, "Statistical multipath propagation modeling for broadband wireless systems," IEICE Transactions on Communications, vol. E90-B, no. 3, pp. 468-484, 2007. 


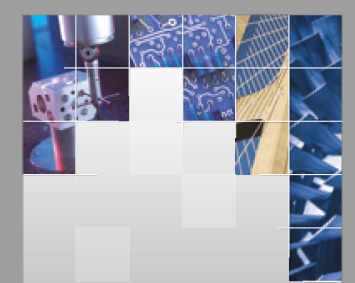

\section{Enfincering}
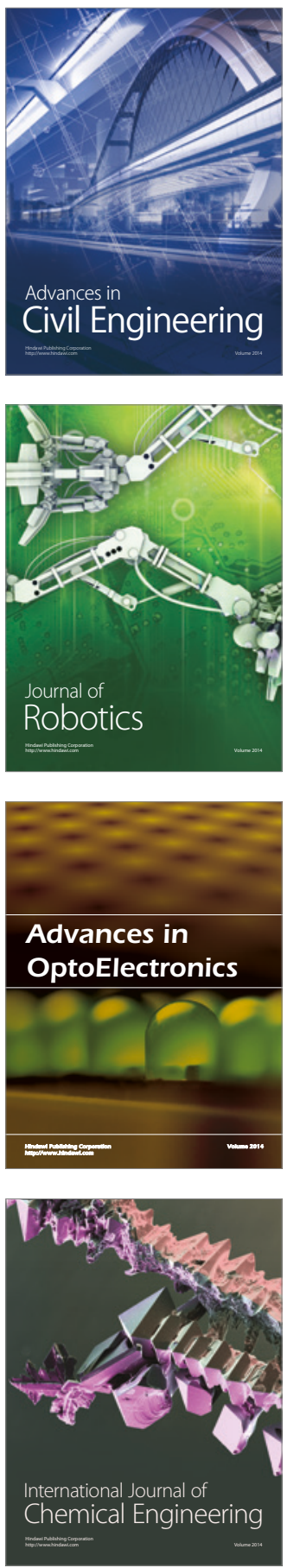

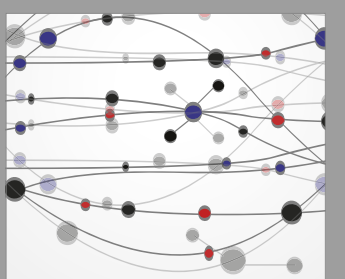

The Scientific World Journal

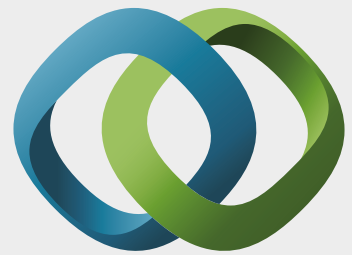

\section{Hindawi}

Submit your manuscripts at

https://www.hindawi.com
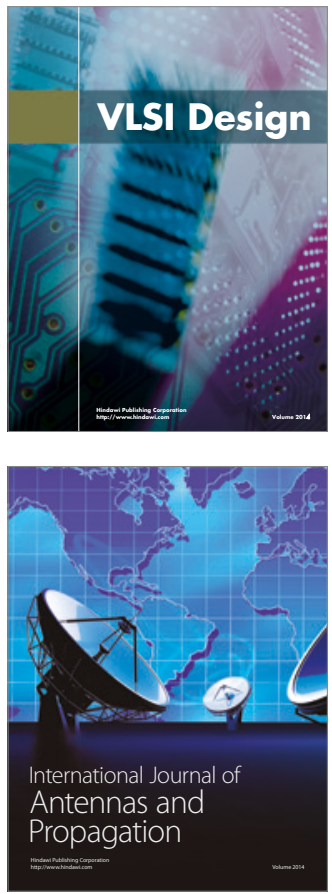

\section{Rotating}

Machinery
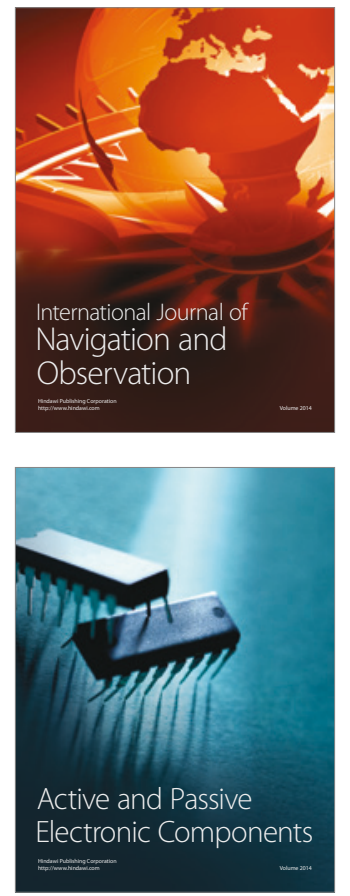
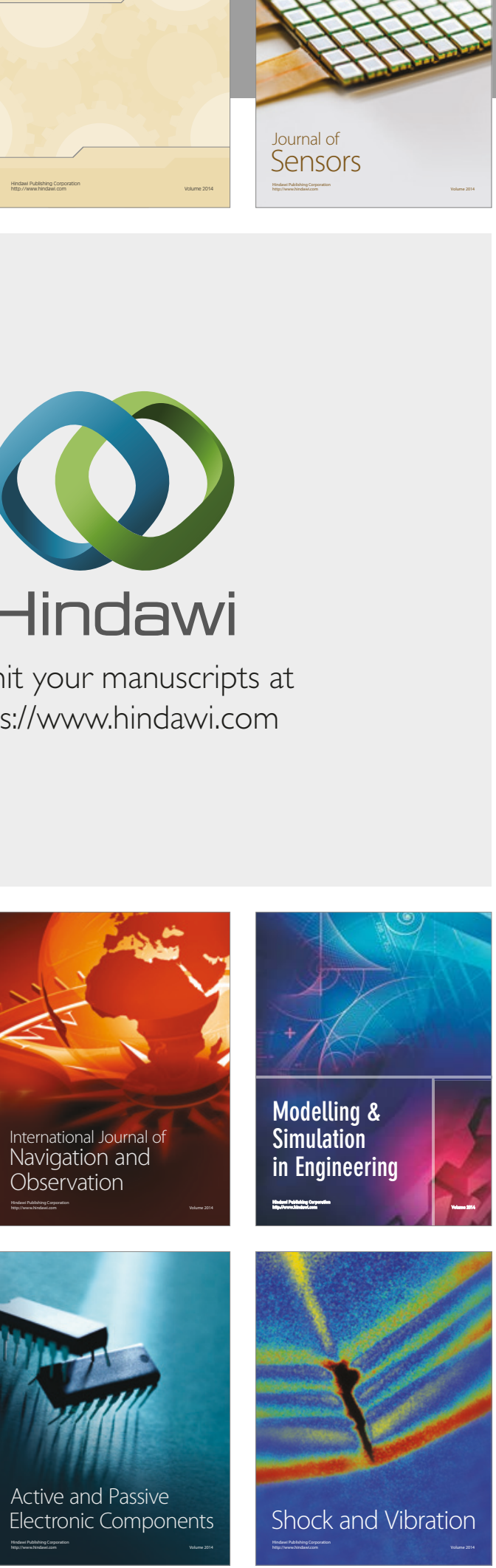
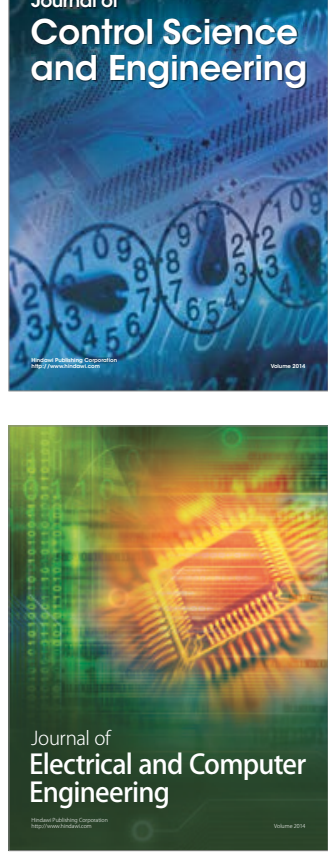

Distributed

Journal of

Control Science

and Engineering
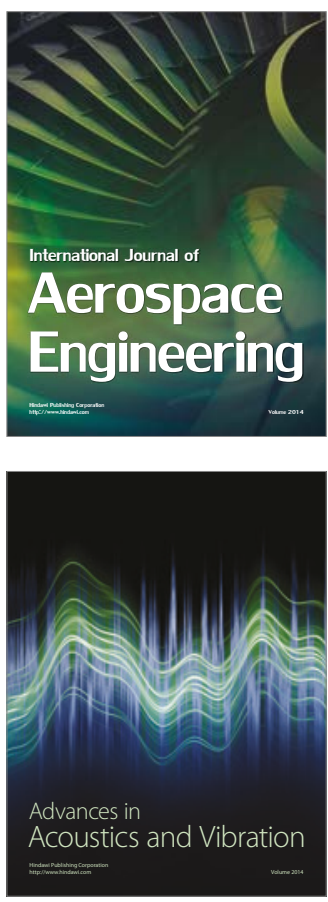

Sensor Networks 